NASA/CR-2000-210108

ICASE Report No. 2000-18

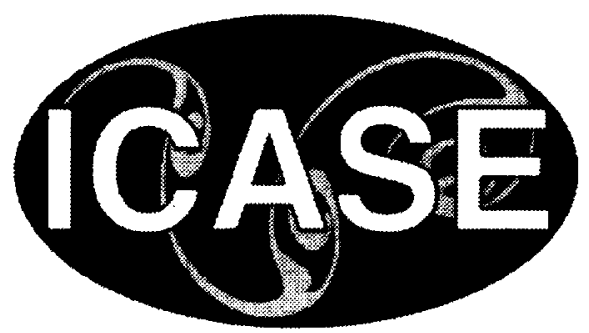

\title{
Improving Jet Reactor Configuration for Production of Carbon Nanotubes
}

Alex Povitsky

ICASE, Hampton, Virginia

Institute for Computer Applications in Science and Engineering

NASA Langley Research Center

Hampton, VA

Operated by Universities Space Research Association

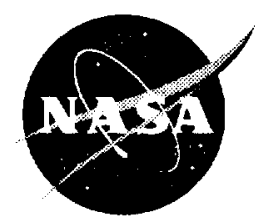

National Aeronautics and

Space Administration

Langley Research Center

Hampton, Virginia 23681-2199
Prepared for Langley Research Center under Contract NAS1-97046

April 2000 
Available from the following:

NASA Center for AeroSpace Information (CASI) 7121 Standard Drive

Hanover, MD $21076-1320$

$(301) 621-0.390$
National Technical Information Service (NTIS) 528.5 Port Royal Road

Springfield, VA 22161-2171

(703) $487-4650$ 


\title{
IMPROVING JET REACTOR CONFIGURATION FOR PRODUCTION OF CARBON NANOTUBES
}

\author{
ALEX POVITSKY*
}

\begin{abstract}
The jet mixing reactor has been proposed for the industrial production of fullerene carbon nanotubes. Here we study the flowfield of this reactor using the SIMPLER algorithm. Hot peripheral jets are used to enhance heating of the central jet by mixing with the ambiance of reactor. Numerous configurations of peripheral jets with various number of jets, distance between nozzles, angles between the central jet and a peripheral jet, and twisted configuration of nozzles are considered. Unlike the previous studies of jet mixing, the optimal configuration of peripheral jets produces strong non-uniformity of the central jet in a cross-section. The geometrical shape of reactor is designed to obtain a uniform temperature of a catalyst.
\end{abstract}

Key words. jet mixing, SIMPLER, jet heating, turbulent jets, nanotube production, fullerenes

Subject classification. Fluid Mechanics

1. Introduction. The $C_{60}$ molecules combined in nanotubes have unusual and very useful electronic and mechanical properties [7], [9]. However, the mass production of nanotubes still presents a challenge. This report describes the CFD modeling and optimization of a jet mixing reactor for such a production. The single-walled carbon nanotubes may be produced from $\mathrm{CO}$ with the help of a catalyst. Catalyst particles get into the reaction chamber via the central $C O$ jet. The particles must be heated from $200 C$ (central jet temperature) to temperatures close to $1000 \mathrm{C}$ (reactor temperature) as rapidly as possible, since slowly heated catalytic particles quickly loose their catalytic ability.

The mixing reactor studied here consists of an arrangement of jets designed to heat-up catalytic particles carried by a turbulent $C O$ jet issuing from a round nozzle. This jet entrains the hot gas within the reactor cavity and heats the jet material. However, this heating is considered too slow to produce a significant amount of carbon nanotubes. To increase mixing, hot turbulent jets issuing from several nozzles surrounding the central nozzle are used. These jets are inclined to the central jet to further increase the mixing rate. The temperature of the hot jets is equal to the average reactor temperature.

The distance between the central and the peripheral nozzles, the number of peripheral jets, and the angle between the central jet and a peripheral jet are taken as optimization parameters. Figure 1 shows a typical jet interaction geometry.

The behavior of a single jet in a static ambiance, the influence of the density difference between the jet and surrounding gas, the effects of co-flowing round jets, jet rotation, and the development of jets in a crossflow stream have been extensively studied [1], [2] and results are summarized as empirical formulas, confirmed by experiments and computations. However, the three-dimensional mixing of jets at high intersection angle $\alpha$ includes mutual dependence of the above factors. Therefore, a detailed CFD investigation and discussion of the physics of interacting jets is needed for the optimization of the reactor.

Holdeman ot al. [4] studied mixing of a set of side jets with a flow in a cylindrical duct and obtained the optimal number of jets for a given jet-to-mainstream momentum flux ratio (or mass flux ratio). In this

\footnotetext{
+Senior Staff Scientist, ICASE, NASA Langley Research Center, Hamptom, VA 23681-2199 (e-mail: aeralpowicase.edu). This research was supported by the National Aeronautics and Space Administration under NASA Contract No. NAS1-97046 while the author was in residence at the Institute for Computer Applications in Science and Engineering (ICASE), NASA Langley Research Center, Hampton, VA 23681-2199.
} 
work, they consider a confined and uniform central flow in a duct, whereas in our reactor a cold central jet is injected into a hot ambient gas. In the current study, the size of the reactor cross-section is larger than that of the central nozzle, therefore, the central jet is more similar to a free jet than to a duct flow. Thus, a peripheral jet should not be mixed with the reactor hot gas. Instead, the peripheral jet must reach the central jet keeping its axial velocity as large as possible and then cause the rapid mixing of the central jet with the ambiance. To achieve this goal, the strongest possible peripheral jets are used. If the total mass flux of peripheral jets is fixed, we show that the minimum number of peripheral jets ensures fast mixing of the central jet with the reactor gas. Our calculations show that two peripheral jets in the $(x, z)$ plane cause the central jet to spread out in the $(y, z)$ plane leading to the fast heating of the cold central jet by mixing with hot reactor gas.

To further increase the mixing of the central jet, the peripheral jet nozzles were drilled off the $z$ axis to ('ause rotation of the central jet. Aerodynamic twisting has been exploited in energy technology [6] to increase turbulent transport. However when heating is to take place at short axial distances, a nozzle arrangement with non-zero total angular momentum of peripheral jets is more advantageous to the basic configuration due to the diagonal elongation of the central jet cross-section than due to its rotation.

For cases where $\alpha$ is greater than $90^{\circ}$, the nozzles are installed on the side walls of the reactor.

The requirements of higher $\alpha$, short distance between a peripheral jet nozzle and its intersection with the central jet, and have enough space in a cross-section to spread the central jet lead to optimization of reactor shape in this study. In our future work, we shall consider such an optimization for non-Cartesian geometry of reactor.

The paper is organized as follows. In Section 2, we conduct a study for the optimal angle $\alpha$, based on empirical expressions for jets in cross-flow. This is considered as a preliminary estimation before the CFD aualysis. In Section 3, we present a CFD framework for our numerical analysis. In Section 4, we study the jets' interaction munerically in order to define the optimal values of afore-mentioned parameters of the jet reactor. In Section 5, we explore a jet arrangement with non-zero total angular momentum leading to aerodynamic twisting of the central flow, and the counter-flow jets' arrangement where the nozzles are drilled through the side walls.

2. Simplified model of mixing of jets. Downstream of the nozzles, the injected cold and hot jets are assumed to develop as free jets until they intersect. We assume that these jets intersect at a single point at the centerline. After the intersection, the central jet axis remains straight due to the symmetric arrangement of the peripheral jets, whereas each of the hot jets is deflected from its original direction by the cross flow of the central jet.

The goal is to ensure fast heating of a central cold jet by peripheral jets. The external part of the central jet has been heated by mixing with the hot ambiance before the intersection with peripheral jets. The problem is to heat up the area of the central jet near the centerline. Therefore, we consider a penetration length (i.e., axial distance between beginning of the jet interaction and the intersection point of jets' axis) as a reasonable indicator of fast heating. We investigate the penetration length as function of the jet intersection angle $\alpha$.

For a round turbulent jet, the centerline velocity distribution is given by [1]

$$
u_{m}=10.7 u_{0} \frac{r_{0}}{x+x_{0}}, \text { if } x>x_{i}
$$

and the distribution of velocity in a cross-section is as follows

$$
u_{, r}=u_{m}\left(1-3 \eta^{2}+2 \eta^{3}\right)
$$


where $\eta=y / b, b=\left(x+x_{0}\right) \tan \beta, \tan \beta=0.22, x_{0}=2.06 r_{0}, x_{i}=8.95 r_{0}, u_{m}$ is the local velocity at the jet axis, $x_{i}$ is the length of initial part of the jet, $u_{0}$ is the jet velocity $(x=0), r_{0}$ is the jet radius, and $\beta$ is the jet expansion angle.

Eqs (1) and (2) are used to compute the jet profiles prior to their interaction.

Demuren [2] uses an empirical model for the trajectory of single turbulent jet injected normally into a cross flow

$$
\frac{y}{d_{f f f}}=a q^{b}\left(\frac{x}{d_{e f f}}\right)^{c}
$$

where $\frac{y}{d_{e f f}}, \frac{x}{d_{e f f}}$ are coordinates of the jet centerline, the empirical coefficients are chosen as $a=0.85$, $b=0.47$, and $c=0.36[2]$, the momentum flux ratio $q=\frac{\rho_{m}}{\rho_{x}}\left(\frac{u_{m}}{u_{x}}\right)^{2}$, index $\infty$ denotes cross flow, and index $m$ denotes parameters of the impinging jet at point $\mathrm{A}$.

In our case the central cold jet remains straight due to the symmetric orientation of heating jets. Thus, we can consider the cold jet as a cross flow. However, the central jet is not equivalent to a uniform cross flow. Thus the linear Taylor expansion of the above expression is used to compute the coordinates of the axis of the peripheral jet at discrete points $(y(i), x(i))$ :

$$
\frac{y}{d_{c f f}}(i+1)=\frac{y}{d_{t f f}}(i)+a c q^{b}\left(\frac{x}{d_{\epsilon f f}}\right)^{c-1} \text { (i) }\left(\frac{x}{d_{f f f}}(i+1)-\frac{x}{d_{c f f}}(i)\right) .
$$

where $q=q\left(u_{\infty}(i), \rho_{\infty}(i)\right)$.

In the above equation, any of the heating jets is assumed to be a circular turbulent jet injected normally into the cross flow. Peripheral jets are no longer uniform while they hit the central jet. The effective diameter of a peripheral jet before it impinges into the central jet is not defined. A reasonable guess is $d_{e f f}=d_{0}$. That suggests consideration of a stream-line tube with the above mentioned diameter. To compute $q$, only the $y$ - component of a peripheral jet is taken into account. The computations of jet trajectory start at point $\mathrm{A}$ and last until the jet intersection at point I (see Figure 1).

The optimal mixing of jets is assumed to be the case where the peripheral jet reaches the centerline of the central jet as fast as possible, i.e. deviation from its initial direction is small. Clearly, this is not the case for small $\alpha$, where jets are almost parallel. Even for large $\alpha$, the dense central jet deviates light peripheral jets.

Figure 2 shows the penetration length, normalized by the orifice radius, versus $\alpha$. These computations lead to $\alpha=90^{\circ}$ which corresponds to the minimum penetration length. However, one should take into account the large hydraulic resistance that occurs for large $\alpha$ and consider the slope of the curve in Figure 2 rather than its minimum value. The slope is large for $a<50^{\circ}$ and small for larger values of $\alpha$. Thus, taking $\alpha \approx 50^{\circ}$ is a reasonable compromise between speedy heating and complications of large values of $\alpha$.

This section offers useful but preliminary analysis of jets' mixing. The CFD results (Sections 4 and 5) show that the central jet develops strong angular non-uniformity in the $(y, z)$ plane. Obviously; this important process in mixing is not taken into account here. Also, the CFD results supply us with a flowfield temperature distribution which is a direct measure of the quality of heating.

\section{CFD model.}

3.1. Governing equations. The Navier-Stokes equations of gas dynamics with an appropriate model of turbulence describe the mixing of jets. The system of transport equations is given by

$$
\sum_{i=1}^{i=3} \frac{\partial}{\partial x_{i}}\left(\Gamma \frac{\partial F}{\partial x_{i}}\right)-\sum_{i=1}^{i=3} \frac{\partial\left(\rho U_{i} F\right)}{\partial x_{i}}+S_{F^{\prime}}=0,
$$


where $x_{1}, x_{2}$, and $x_{3}$ are Cartesian coordinates, $F=U_{1}, U_{2}, U_{3}, k, \epsilon, C, T$ are the main variables, $U_{1}, U_{2}, U_{3}$ are velocity components, $k$ and $\epsilon$ are kinetic energy of the turbulence and the turbulent dissipation, $C$ is the mass concentration of material in the central (cold) jet, $T$ is the temperature, $S_{F}$ is the source term, and $\Gamma$ is the transport coefficient. The Standard $k-\epsilon$ model [8] is used in this study to predict turbulent transport when the turbulent viscosity and transport coefficient are given by

$$
\begin{gathered}
\Gamma=\frac{\mu_{e f f}}{\operatorname{Pr}_{F}}, \\
\mu_{e f f}=c_{\mu} \rho k^{2} / \epsilon,
\end{gathered}
$$

where $c_{\mu}=0.09$ is the coefficient of the $k-\epsilon$ model of turbulence, and $\operatorname{Pr}_{F}$ is the Prandtl number for variable $F$.

TABLE 1

Prondtl numbers and source terms for governing equations

\begin{tabular}{|c|c|c|}
\hline Variable & Prandtl number & Source Term \\
\hline$U_{i}$ & 1. & $-\frac{\partial P}{\partial x_{i}}$ \\
$C$ & .75 & - \\
$T$ & .9 & - \\
$k$ & 1. & $Q-\epsilon \rho$ \\
$\epsilon$ & 1.3 & $\left(c_{1} Q-c_{2} \epsilon \rho\right) \epsilon / k$ \\
\hline
\end{tabular}

In Table $1, c_{1}=1.44$ and $c_{2}=1.92$ are coefficients of the $k-\epsilon$ model of turbulence, and $Q$ is the rate of turbulence energy generation:

$$
Q=\mu_{e f f} \sum_{i, j=1}^{i, j=3}\left(\frac{\partial U_{i}}{\partial x_{j}}\right)\left(\frac{\partial U_{i}}{\partial x_{j}}+\frac{\partial U_{j}}{\partial x_{i}}\right)-\frac{2}{3} k \rho \sum_{i=1}^{i=3}\left(\frac{\partial U_{i}}{\partial x_{i}}\right) .
$$

For the special case where Mach number $M<0.3$, the density is assumed to be independent of pressure. For mixing of chemically inert jets, Eq. (5), with zero source term, is solved for the concentration $C$ of material in the cold jet. To satisfy the boundary conditions, the concentration $C$ is set equal to unity at the central nozzle and equal to zero at the peripheral nozzles. Using the known concentration field, the local density is computed by

$$
\rho=C \rho_{\text {cold }}+(1-C) \rho_{h o t}
$$

where $\rho_{c o l d}$ and $\rho_{\text {hot }}$ are densities of the central and peripheral jets.

The catalyst particles considered are small (less than $20 \mu$ ) in terms of their size and mass, therefore, they are assumed to move with zero velocity relative to the gas. Thus, the same concentration field $C$ shows the spatial distribution of catalyst particles.

The temperature field is computed by $\mathrm{E}_{\mathrm{q}}$. (5) with zero source term, where the boundary conditions are similar to those for the concentration field. Still, the coefficients of the PDE for temperature are different from those for concentration as their Prandtl numbers are different. Note that the temperature field does not affect other governing equations.

3.2. Numerical algorithm. We discretize the governing PDEs (5) using a finite volume method and a structured numerical grid. The current version of the developed computer code includes discretization on 
uniform or non-uniform grids in Cartesian coordinates. For simplicity, we describe the uniform grid version here. The basic grid is used for discretization of $T, C, P, K$, and $\epsilon$ governing variables:

$$
\begin{aligned}
& x_{1}(i)=(i-1) h_{1}, i=1, \ldots, N_{1}, \\
& x_{2}(j)=-L_{2}+(j-1) h_{2}, j=1, \ldots, N_{2}, \\
& x_{3}(k)=-L_{3}+(k-1) h_{3}, k=1, \ldots, N_{3},
\end{aligned}
$$

where $h_{1}=L_{1} /\left(N_{1}-1\right), h_{2}=2 L_{2} /\left(N_{2}-1\right), h_{3}=2 L_{3} /\left(N_{3}-1\right)$ are grid steps, and $L_{1} \times 2 L_{2} \times 2 L_{3}$ is the computational volume. The symmetry of solution is one indicator of the correctness of computer code, therefore, we do not halve the computational domain by imposing the symmetric boundary conditions unless it is stated otherwise. Staggered grids are used for discretization of velocity components:

$$
\begin{aligned}
x u(i) & =0.5\left(x_{1}(i)+x_{1}(i-1)\right), i=2, \ldots, N_{1} \\
x v(j) & =0.5\left(x_{2}(j)+x_{2}(j-1)\right), j=2, \ldots, N_{2} \\
x w(k) & =0.5\left(x_{3}(k)+x_{3}(k-1)\right), \quad k=2, \ldots, N_{3},
\end{aligned}
$$

where $x u(1)=x_{1}(1)-0.5 h_{1}, x v(1)=x_{2}(1)-0.5 h_{2}, x u(1)=x_{3}(1)-0.5 h_{3}, x u\left(N_{1}+1\right)=x_{1}\left(N_{1}\right)+$ $0.5 h_{1}, x v\left(N_{2}+1\right)=x_{2}\left(N_{2}\right)+0.5 h_{2}, x w\left(N_{3}+1\right)=x_{3}\left(N_{3}\right)+0.5 h_{3}$. Grids $\left\{x u, x_{2}, x_{3}\right\},\left\{x_{1}, x v, x_{3}\right\}$, and $\left\{x_{1}, x_{2}, x w\right\}$ are used for discretization of $U_{1}, U_{2}$, and $U_{3}$, respectively. Basic and staggered grids in a 2 D section are shown in Figure 3. The finite-volume method for discretization of Eq. (5) on any of the described above numerical grids leads to the following set of equations with respect to unknown values of $F$ in neighboring grid points:

$$
a_{P} F_{P}=a_{N} F_{N}+a_{S} F_{S}+a_{E} F_{E}+a_{W} F_{W}+a_{T} F_{T}+a_{B} F_{B}+S_{F},
$$

where

$$
\begin{aligned}
a_{P}= & a_{E}+a_{W}+a_{N}+a_{S}+a_{T}+a_{B}+ \\
& \frac{\left(\rho U_{1}\right)_{t}-\left(\rho U_{1}\right)_{w}}{x_{1, t}-x_{1, w}}+\frac{\left(\rho U_{2}\right)_{n}-\left(\rho U_{2}\right)_{s}}{x_{2, n}-x_{2 .,}}+\frac{\left(\rho U_{3}\right)_{t}-\left(\rho U_{3}\right)_{b}}{x_{3, t}-x_{3, b}} ; \\
a_{E}= & \frac{1}{x_{1, e}-x_{1, w}} \max \left(-\left(\rho U_{1}\right)_{e} ; \frac{\Gamma}{x_{1, E}-x_{1, P}}-0.5\left(\rho U_{1}\right)_{e} ; 0\right) ; \\
a_{W}= & \frac{1}{x_{1, e}-x_{1, u}} \max \left(\left(\rho U_{1}\right)_{u} ; \frac{\Gamma}{x_{1, P}-x_{1, W}}+0.5\left(\rho U_{1}\right)_{w} ; 0\right) .
\end{aligned}
$$

Here indices $E, W, N, S, T, B$ correspond to the immediate neighbors of the point $P$ (see Figure 4 ). Velocities in corresponding directions are computed at mid-points $e, w, n, s, t, b$. Formulas for coefficients $a_{E}, a_{W}, a_{T}, a_{B}$ are similar to above formulas for coefficients $a_{N}$ and $a_{S}$. This form of discrete coefficients implies a central difference second-order scheme while $|P \ell| \leq 2$ and a first order upwind scheme otherwise [5], [3], where $P e=\rho U \Delta x / \Gamma$. To compute coefficients (12), the turbulent viscosity and density at the point $e$ are calculated as the arithmetic average of its values at points $E$ and $P$. Eqs. (11) with coefficients similar to Eqs. (12) are used to compute velocities on staggered grids.

For computations of the pressure field and correction of velocities, that are made to satisfy the continuity equation, Patankar's SIMPLER (Semi-Implicit Method for Pressure-Linked Equations Revised) is used [5]. The reduced formulas (11) for velocities lead to the following expressions connecting the velocity and pressure corrections:

$$
U_{e}^{\prime}=-\frac{P_{E}^{\prime}-P_{P}^{\prime}}{\left(x_{1 . E}-x_{1 . P}\right) a_{e}},
$$


where $a_{e}$ is the coefficient $a_{P}$ for velocity $U_{1}$. The SIMPLE-type algorithms substitute the above formulas in the continuity equation to form Eq. (11) for the pressure correction. The set of discretized equations for pressure correction is given by

$$
b_{P} P_{P^{\prime}}^{\prime}=b_{N} P_{N}^{\prime}+b_{S} P_{S}^{\prime}+b_{E} P_{E}^{\prime}+b_{W} P_{W}^{\prime}+b_{T} P_{T}^{\prime}++b_{B} P_{B}^{\prime}+S_{P^{\prime \prime}}
$$

where

$$
\begin{aligned}
b_{P} & =b_{N}+b_{S}+b_{E}+b_{W}+b_{T}+b_{B} ; \\
b_{N} & =\frac{1}{x_{1, e}-x_{1, u}} \frac{\rho_{c}}{a_{N}\left(x_{1, E}-x_{1, P}\right)} ; \\
b_{S} & =\frac{1}{x_{1, e}-x_{1, u}} \frac{\rho(i-1 / 2)}{a_{S}\left(x_{1, P}-x_{1, W}\right)} ; \\
b & =-\left(\frac{\left(\rho U_{1}\right)_{e}-\left(\rho U_{1}\right)_{u}}{x_{1, e}-x_{1, u}}+\frac{\left(\rho U_{2}\right)_{n}-\left(\rho U_{2}\right)_{s}}{x_{2, e}-x_{2, u}}+\frac{\left(\rho U_{3}\right)_{t}-\left(\rho U_{3}\right)_{b}}{x_{3, t}-x_{3, b}}\right) .
\end{aligned}
$$

After computing the $P^{\prime}$ field, velocity corrections are computed by Eq. (13). SIMPLER employs the pressure-correction equation only for the task of correcting the velocities and not for the computation of the pressure field.

To compute pressure, Eq. (14) is used with coefficients similar to (15), where pseudo-velocities are used instead of velocities [5]. At each external iteration by all governing equations, the Poisson Eqs. (14) for pressure and for pressure correction should be solved up to a high level of convergence to ensure the satisfaction of the continuity equation.

4. Arrangement of nozzles at the reactor's entrance. The reactor is considered as a box with rectangular entrance section, equipped with nozzles, impermeable side walls, and open exit. Physical variables are normalized by the central nozzle radius, the central jet speed and its density at the nozzle.

The cold central jet and hot peripheral jets have initial temperatures $T_{j e t}=200 \mathrm{C}$ and $T_{\mathrm{e} m}=1000 \mathrm{C}$, respectively. In all cases considered, the nozzle gas velocity of a peripheral jet is equal to that of the central jet. In Case 7 (two peripheral parallel jets), the radius of the central orifice and each of peripheral orifices are equal. For a fair comparison, the total mass flux and initial velocity of the peripheral jets are taken the same for all considered configurations of nozzles. Therefore, the radius of a peripheral nozzle is computed by:

$$
r_{p}=r_{p 0} /\left(0.5 N_{j} \cos (\alpha)\right)^{0.5}
$$

where $r_{\mu 0}$ is the radius of a peripheral orifice in Case 7 .

Input data for the considered arrangements of nozzles are gathered in Table 2. In fact, the difference between cases is fully determined by the boundary conditions at the entry section. The boundary conditions at the entry cross-section and a section of the numerical grid in $(y, z)$ plane are shown in Figure 5 .

The quality of heating of the catalyst particles is estimated from the particle temperature distribution at the reactor exit cross-section. This distribution shows the heating rate on average and the uniformity of heating. The height of a histogram column is computed by

$$
h_{i}=\int_{S} \rho U_{1} C d x d y
$$

where $S$ is the subset of the cross-section such as $\theta_{i-1}<\theta<\theta_{i}$, and $\theta=\left(T-T_{j e t}\right) /\left(T_{e n t}-T_{j+t}\right)$. 
'TABI.F 2

Arrangernents of nozzles

\begin{tabular}{|c|c|c|c|}
\hline Case & Distance, $r / r_{c}$ & Angle, $\alpha$ & Number of jets, $N_{j}$ \\
\hline 1 & - & - & single jet \\
2 & 2.0 & 5 & 4 \\
3 & 2.0 & 60 & 4 \\
4 & 10.0 & 60 & 4 \\
5 & 10.0 & 30 & 2 \\
6 & 10.0 & 60 & 2 \\
7 & 10.0 & 0 & 2 \\
\hline
\end{tabular}

The first case corresponds to a single jet heated up by spreading into a hot ambiance. This case shows the basic rate of heating for the simplest arrangement of the reactor (see Figures 6 and 11a). The second and the third cases represent the compact arrangement of four peripheral jets taken as close as possible to the central jet. The former case has almost a parallel arrangement of jets, whereas in the latter case the angle between the central and a peripheral jets is equal to $60^{\circ}$. Some computational flow fields in the $(x, z)$ plane are shown in Figure 7. The co-flowing peripheral and central jets lead to elongation of the high-speed centerline core of the central jet in comparison to the single jet (compare Figures 7 a and 6). This leads to slower mixing of the central jet with the ambiance. Although the co-flowing component of the peripheral jet speed is halved in the latter case, the jet elongation is still noticeable in this case (see Figure 7d). The concentration and temperature fields show the behavior similar to that for velocity (and momentum) fields (see Figure $7 \mathrm{~b}, c, e, f$ ).

In turn, this leads to slow mixing of the central jet with the hot gas and the exit temperature distribution of particles is either lower than that for the single jet (Case 2) or only marginally higher (Case 3) (compare Figure 11a, b, and c).

To reduce the co-flowing effect, the peripheral jet nozzles were placed at some distance from the central jet (Case 4). This leads to a definite increase of the final particle temperatures (compare Figure 11d to Figure 11a). However, the distance between the peripheral jet nozzles and the jets' intersection should be small in order to keep the peripheral jet speed. As it was mentioned in the previous Section, the core velocity of the free jet remains equal to its nozzle velocity while the axial distance from the nozzle is less than $10 r_{0}-12 r_{0}$ and then it decreases proportional to this distance.

To increase the mass and momentum flux of a single peripheral jet, configurations with two peripheral jets are considered (Cases $7,5,6$ ) and computational fields are shown in corresponding Figures 8, 9, and 10. In Case 7 (parallel jets), the inclination of the peripheral jets towards the central jet occurs only due to the entrainment of these light hot jets by the dense central cold jet. The difference between the jets' plane and the middle plane (compare Figure 8a,c to b,d) is slight and the quality of heating is close to that for Case 1. Therefore, the entrainment of light (hot) jets by the dense (cold) jet should not be considered as a major factor of the cold jet heating.

The angular variation of field isolines in $(y, z)$ plane becomes considerably more prominent for initially inclined jets (Cases 5 and 6 ). The central jet narrows in the plane $(x, z)$, where the peripheral jets hit it. and spreads aside in the plane $(x, y)$ (see Figures 9 and 10). As opposed to the previous cases, the heated particles occupy the entire exit cross-section (see Figures $9 \mathrm{~d}$ and 10d). The quality of heating is best in Case 6 and second best in Case 5 (see Figure 11e,f). 
5. Offset and side-wall jet configurations. In this section we consider peripheral jets with nonzero total angular momentum (Case 8), and peripheral jets with $\alpha>90^{\circ}$ (Case 9) (see Table 3). Two peripheral jets are taken here according to results shown in the previous Section. In order to produce

TABLE 3

Offset and side-wall configurations of peripheral nozzles

\begin{tabular}{|c|c|c|c|}
\hline Case & Coordinates of nozzles $(x, y, z)$ & Angle, $\alpha$ & Cross-section $(y, z)$ \\
\hline 8 & $\left(0, \pm r_{0}, \pm 10 r_{0}\right)$ & $60^{*}$ & $20 r_{0} \times 20 r_{0}$ \\
9 & $\left(17.3 r_{0}, 0, \pm 20 r_{0}\right)$ & 120 & $20 r_{0} \times 20 r_{0}$ \\
10 & $\left(17.3 r_{0}, 0, \pm 10 r_{0}\right)$ & 120 & $20 r_{0} \times 10 r_{0}$ \\
\hline
\end{tabular}

angular momentum in the central flow, the peripheral nozzles are drilled at distance $\pm 1 r_{0}$ from the $z$ axis. In this case, the angle $\alpha$ is the angle between a peripheral jet and a horizontal plane that includes the central cold jet. This configuration of jets leads to slightly better mixing than that in Case 6 (see Figure 12b). The reason for better mixing is the resulting cross-sectional elongation of the central jet along the diagonal of the cross-section (see Figure 12a). as opposed to elongation in the $y$ direction as in Case 6 . The simple geometric observation that the square diagonal is $\sqrt{2}$ times larger than the square edge explains the difference in mixing between Cases 8 and 6 .

For $x \leq 30 r_{0}$, the rotation of the central jet remains small and cannot lead to a substantial increase of jet mixing.

Next, we investigate Case 9 , where the jet intersection angle $\alpha=120^{\circ}$ between the central and a peripheral jets. To make it feasible, the peripheral nozzles are drilled through the side wall. For fair comparison to Case 6, the jets are designed to meet at the same axial location. The higher angle between jets causes better mixing, however, this nozzle arrangement increases the distance a peripheral jet travels before intersecting the central jet. From geometric consideration, this distance in Case $\mathbf{9}$ is twice as large as that in Case 6 (compare Figure 13a to Figure 10a). The tradeoff between higher angle of incidence and smaller maximum local peripheral jet velocity may lead to different conclusions about the efficiency of such an arrangement of jets. Here, the temperature distribution of particles is slightly different from that in Case 6 (compare Figure 11f to Figure 13d). In Case 9, the amounts of well-heated particles $(0.90<\theta \leq 0.95)$ and poorly heated particles $(0.7<\theta \leq 0.75)$ increase at the expense of intermediately heated particles.

To achieve a smaller distance between a peripheral jet nozzle and the intersection point (Case 10, Table 3 ), the cross-section of reactor is changed from a square to a rectangle in such a way that its height was halved and its width remains the same as in Case 9. Other parameters in Case 10 remain the same as in Case 9. This cross-sectional shape combines the strength of peripheral jets (as their traveling distance is halved) and provides the available space for spreading the central jet (as the $y$-edge remains unchanged). For these cases we impose symmetric boundary conditions at the symmetry planes and compute a quarter of the computational volume (see Figure 14). The impact of the peripheral jet on the central jet is stronger than that in Case 9 (compare Figure 14a to Figure 13a). Some particles mix with the reactor ambiance upstream of the jets' intersection (Figure 14b) because the strong peripheral jets act as an obstacle to the central jet and create stagnation-type flow at the centerline. The mixing of particles within the reactor downstream of the jets' intersection occurs through the impact of the crosswise component of flow on the side walls and the creation of vortices in the cross-section shown in Figure 14c. Intensive mixing leads to an even heating of particles (see Figure 14d). 
6. Conclusion. Fast mixing and heating of the central jet occurs in cases with strong angular nonuniformity of the merged jet in a cross-section $(y, z)$ and wide-spreading of particles. This appears to be a key process for the aerodynamic mixing of the central jet with the reactor ambiance. Unlike previous studies with high angular uniformity in a cross-section and use of multiple peripheral jets, the maximum strength of each peripheral jet suggests a configuration of two peripheral jets with high intersection angle between the central and a peripheral jets. The optimal distance between peripheral nozzles and the central jet results from the tradeoff between the strength of a peripheral jet while it hits the central jet and the co-flowing effect delaying mixing of the central jet.

Arranging peripheral nozzles in such a way that the total angular momentum of the peripheral jets is non-zero helps to control the direction of spread of the central jet and may increase its mixing. Using counter-flow jets increases the spread-up of the central jet and requires detailed investigation of the reactor geometry.

Further studies will be conducted to investigate the effect of cross-section shape and size on mixing. Possible improvements include variable axial size of cross-section. A small effective diameter of the reactor cross-section, where peripheral jets hit the central jet, provides the strength of peripheral jets, whereas larger reactor cross-section downstream provides the necessary space for elongation of the central jet's crosssection. Perhaps, an elliptical cross-section provides the required elongation length in one direction and a short distance between the peripheral nozzles and the central jet in the other direction. To perform this

study, the wall boundary layer model and the ability to handle complex geometries will be incorporated in the computer code.

\section{REFERENCES}

[1] G. N. ABramovich, The Theory of Turbulent Jets, The MIT Press, Cambridge, MA, 1963.

[2] A. Denuren, Modeling of jets in cross-flow, in Handbook of Fluid Dynamics and Fluid Machinery, J. A. Schetz and A. E. Fuhs, eds., John Wiley, New York, 1996, pp. 787-823.

[3] C. Hinsch, Numerical Computation of Internal and External Flous, Vol. 1: Fundamentals of Numerical Discretizations, John Wiley, Chichester, 1994.

[4] J. D. Holderman e' Al., Mixing of Multiple. Jets With a Confined Subsonic Crossflow in a Cylindrical Duct, NASA Technical Memorandum 107185, NASA Lewis Research Center, 1996. ASME-96-GT482, Prepared for the 41st Gas Turbine and Aeroengine Congress, ASME, Birmingham, UK. June 1996.

[5] S. Patankar, Numerical Heat Transfer and Fluid Flow, Hemisphere Publishing Co., New York, 1980.

[6] A. Povitsky and Y. Goldman, Solid Fuel Gas Turbine and Ash Separation System, Fuel, 75 (1996). pp. $551-559$.

[7] R. SMalley, Discovering the Fullerenes, Nobel Lecture, Center for Nanoscale Science and Technology; Rice University, Houston, Texas, 1996. http://cnst.rice.edu/nobel.html.

[8] D. Whcox, Turbulence Modeling for CFD, DCW Industries, Inc., La Canada, California, 1998.

[9] B. Yakobson and R. Smalley, Fullerene Nanotubes: $C_{1,000,000}$ and Beyond, American Scientist, (JulyAugust 1997), pp. 81116. 


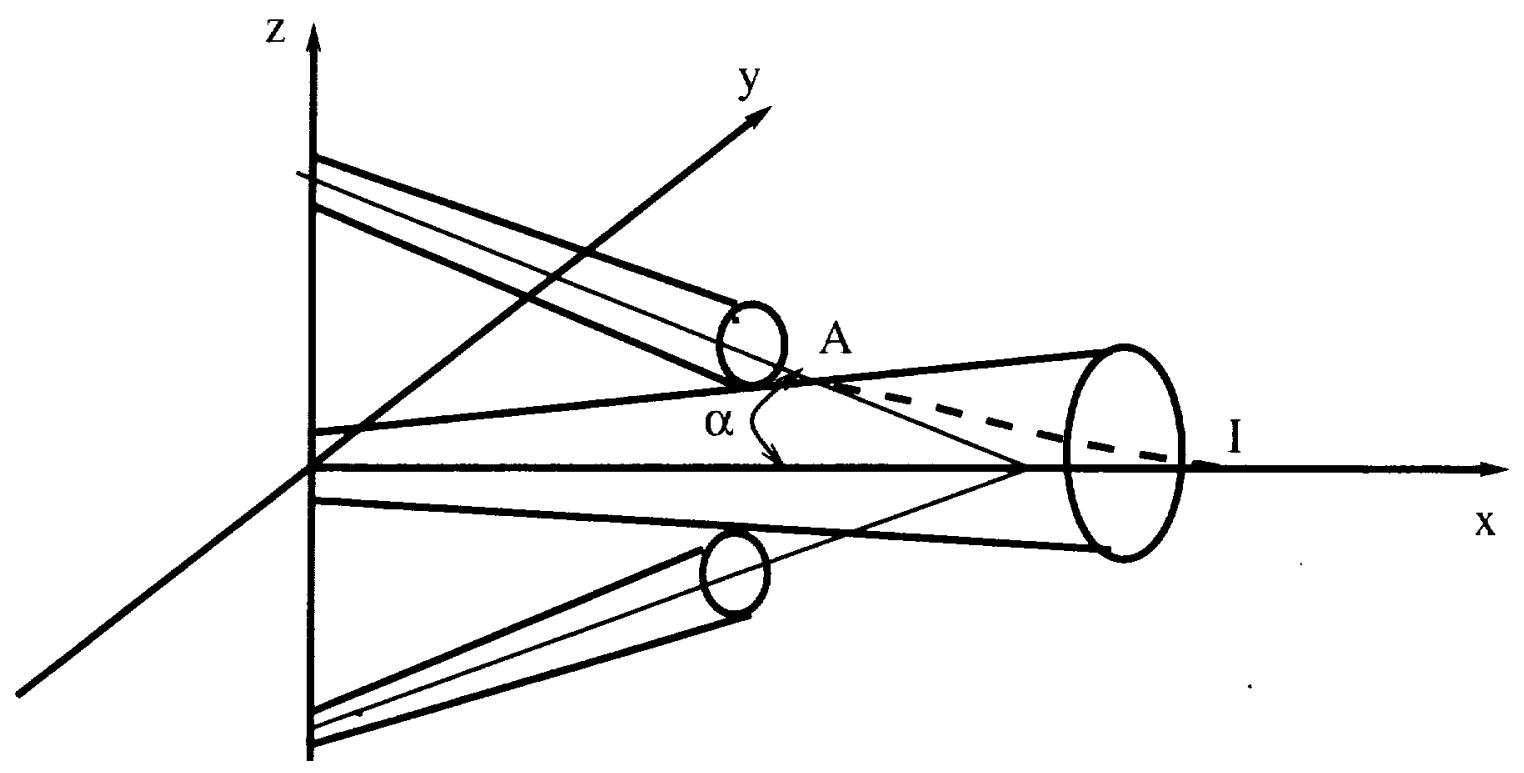

Fli. 1. Typical jet interaction geonetry, jets begin interact at point $A$ and their centerines cross at point I

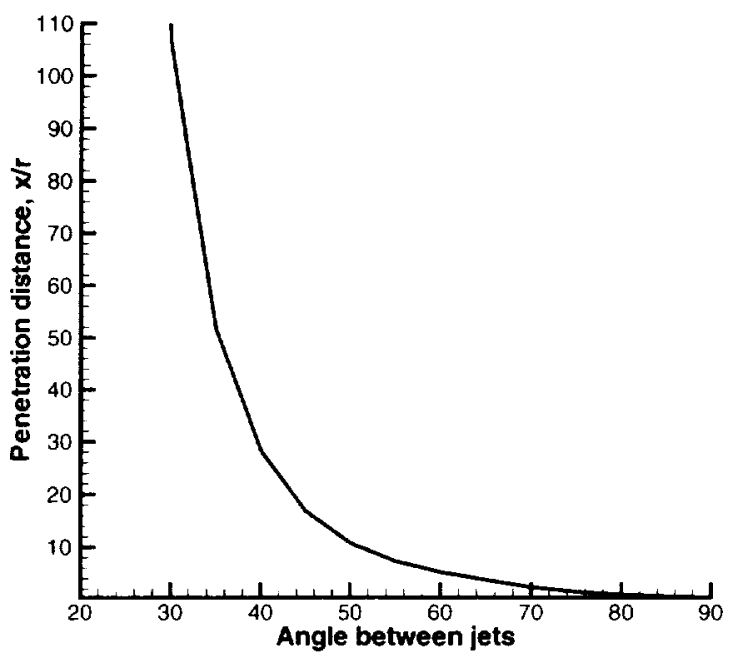

F1G. 2. Penetration length as function of the jet intersection angle 


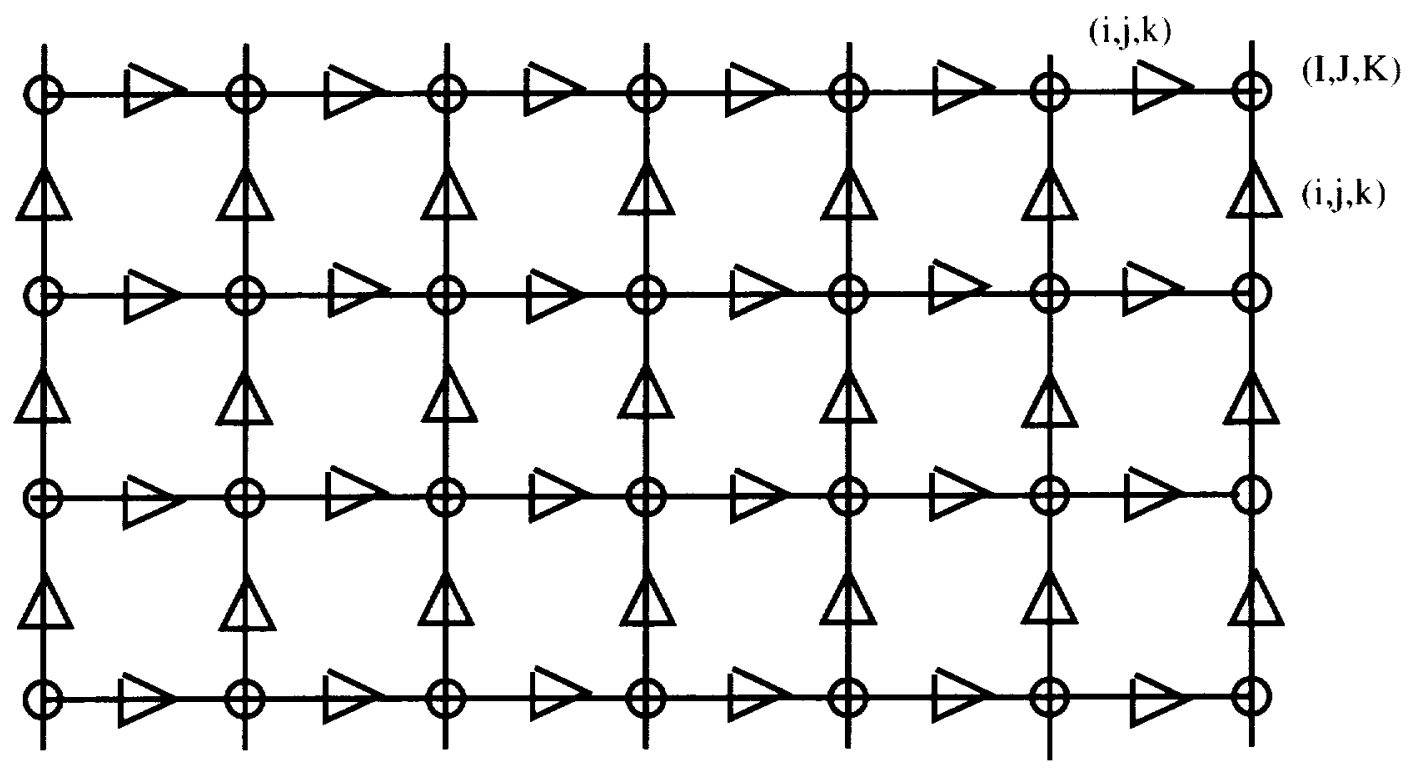

FiG. 3. Staggered grid (2-D $(x, y)$ section), $\bigcirc$ is the numerical grid for $T, C, P, K, c$ fields, $\triangleright$ is the numerical grid for $V_{1}$ and $\triangle$ is the numerical grid for $U_{2}$.

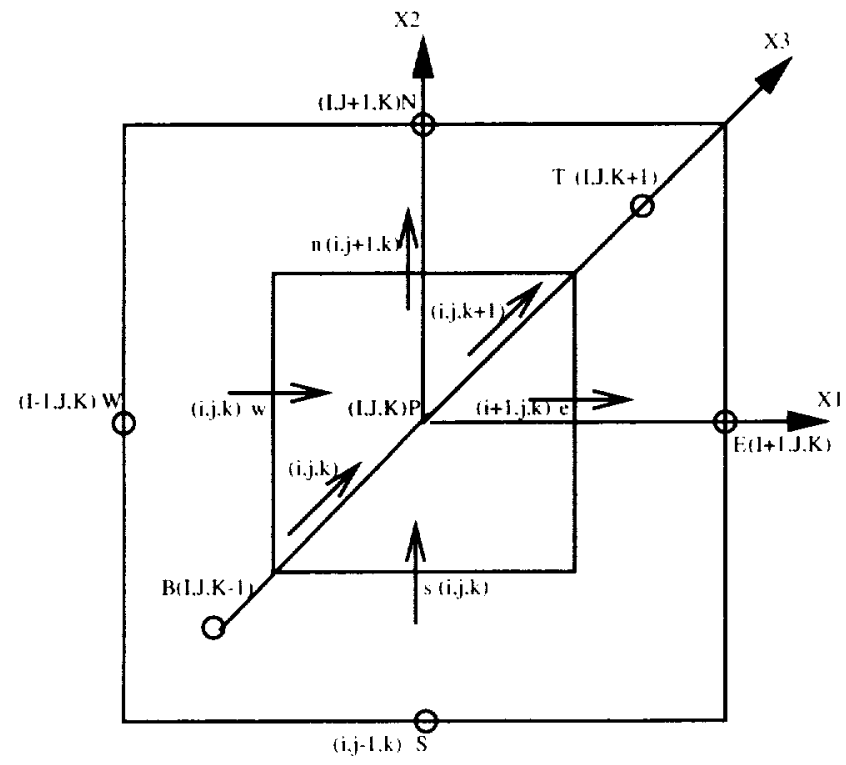

FIG. 4. Control volume, capital letters $(I, J, K)$ denote the basic grid, small letters $(i, j, k)$ denote staggered grids

11 


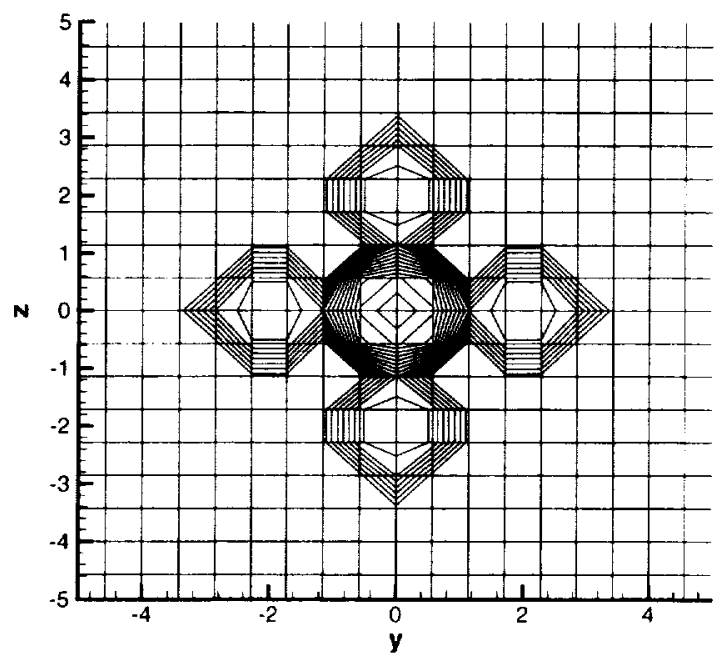

FIG. 5. Fragment of entry cross-section for Case 2

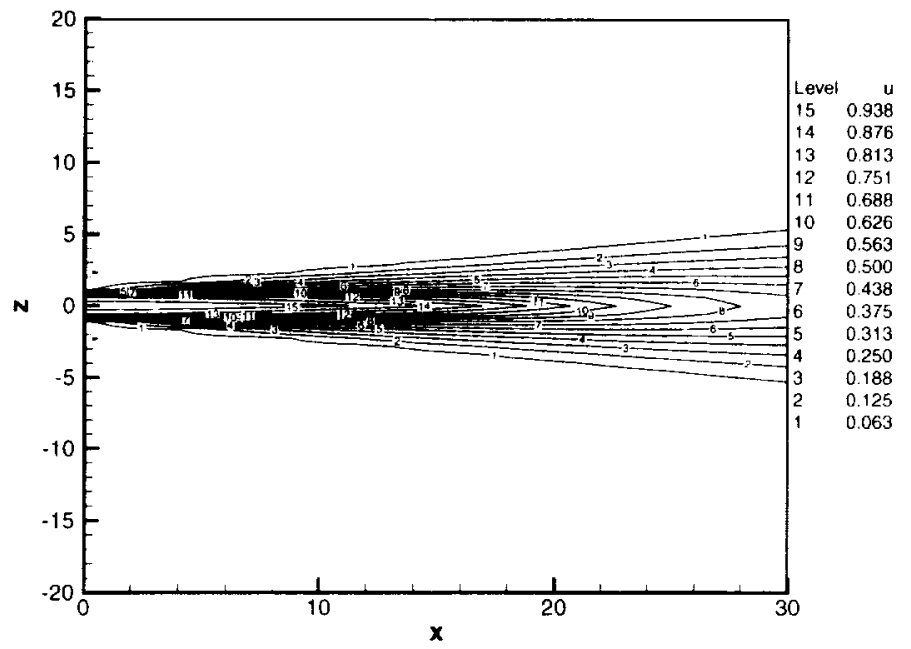

FIG. 6. Velocity isolines of a single cold jet in hot ambiance 


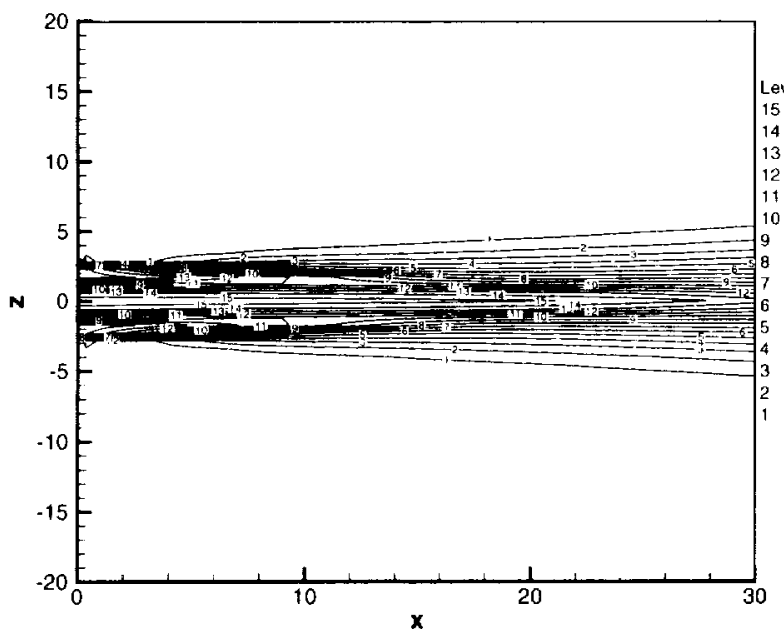

a

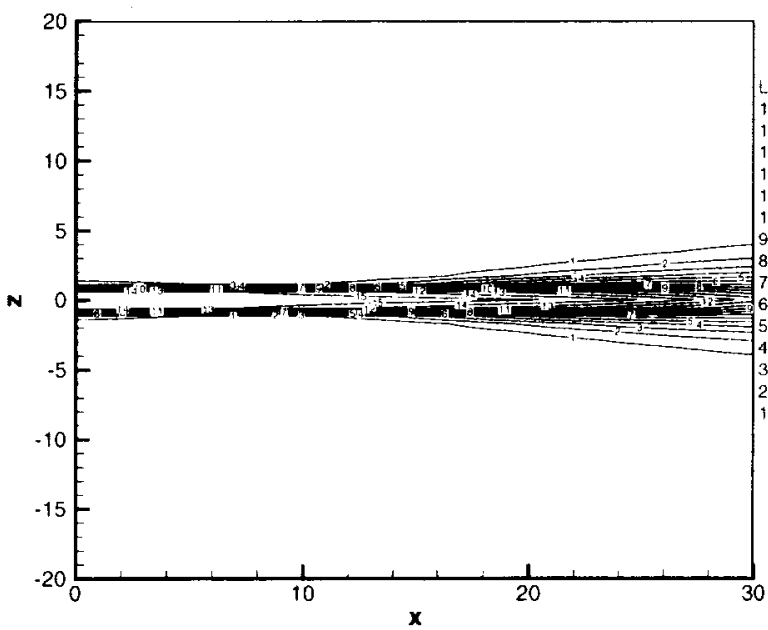

b

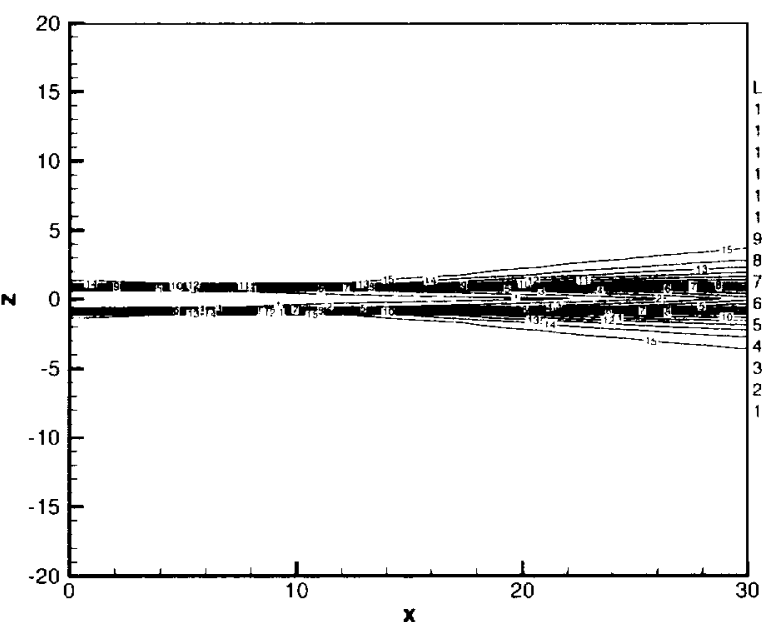

(:

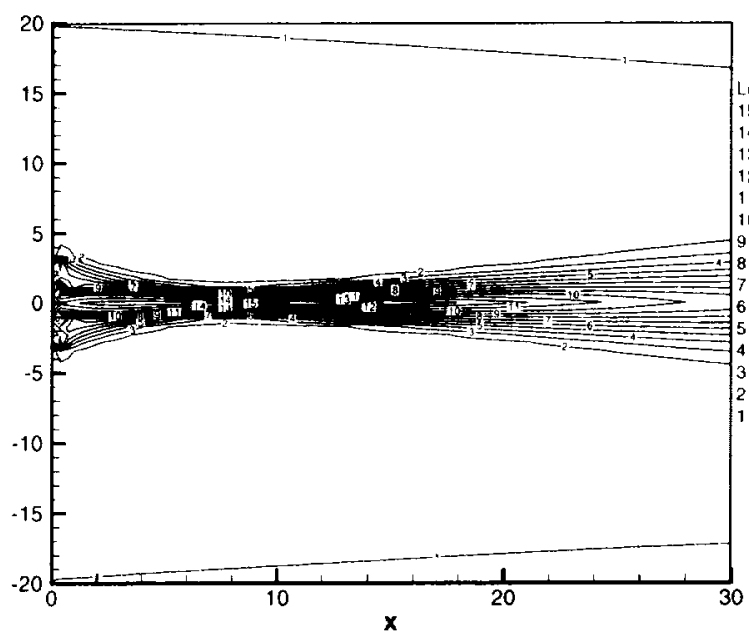

d

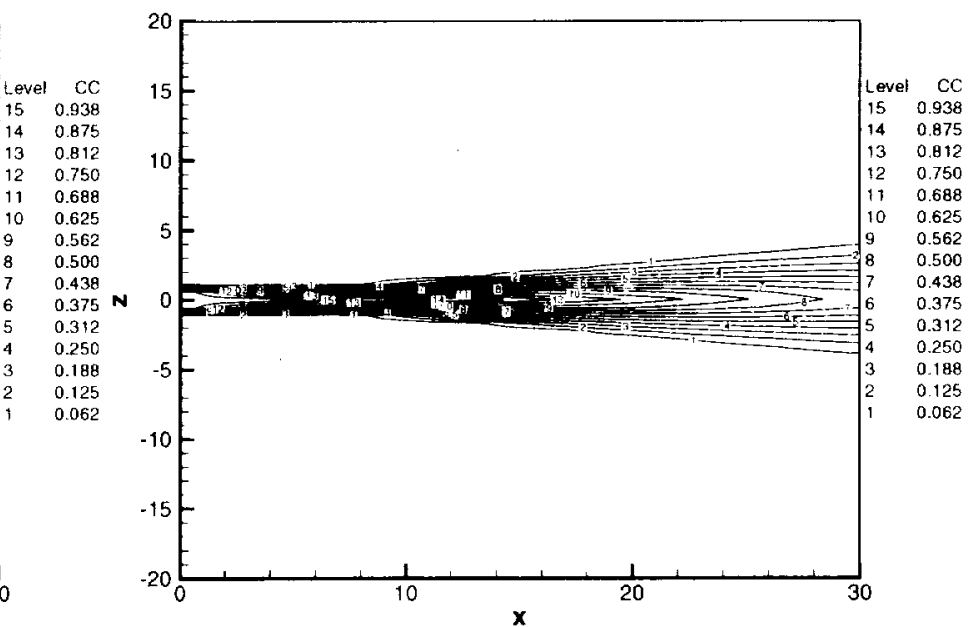

(?

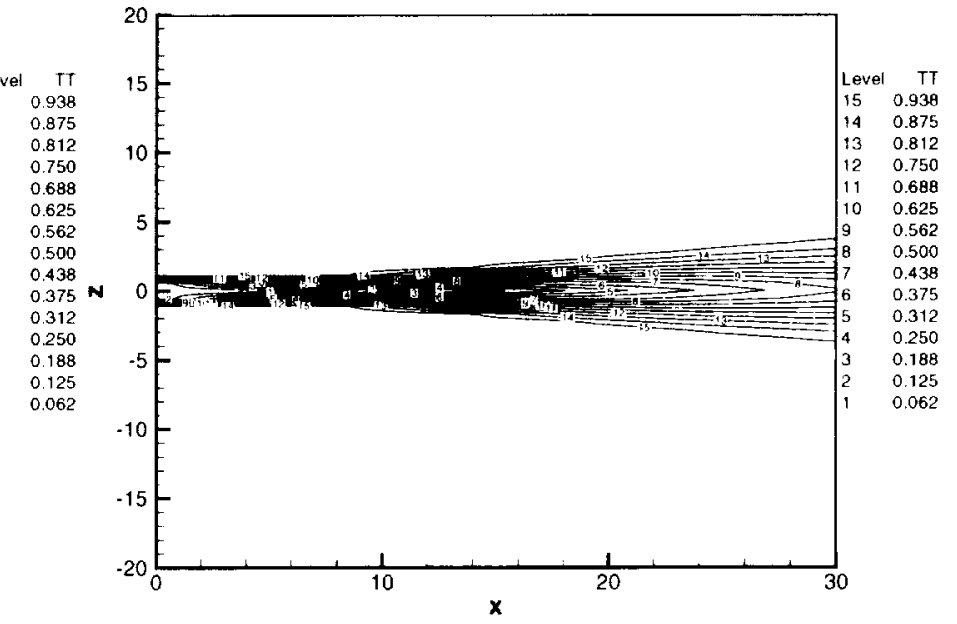

f

Fig. T. Compact configuration of four peripheral nozzles: $\left.a, b, c)-\alpha=5^{\prime \prime}, d, e, f\right)-\alpha=60^{\circ},(a, d)$-axial velocity, (b,e)concentration, $(c, f)$-temperature 


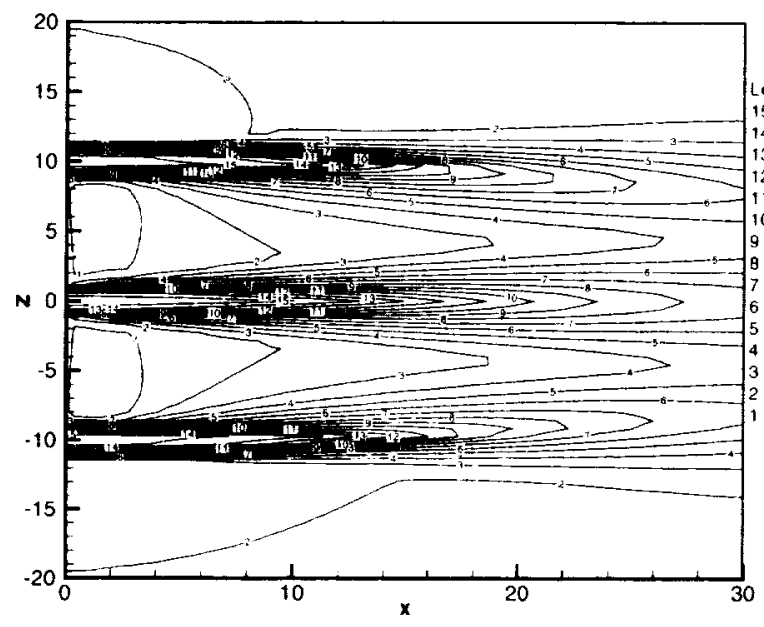

a

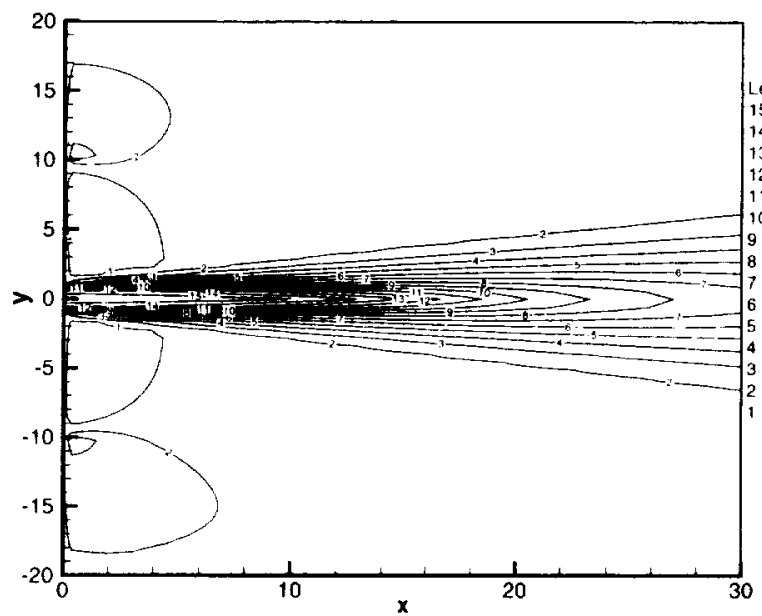

(

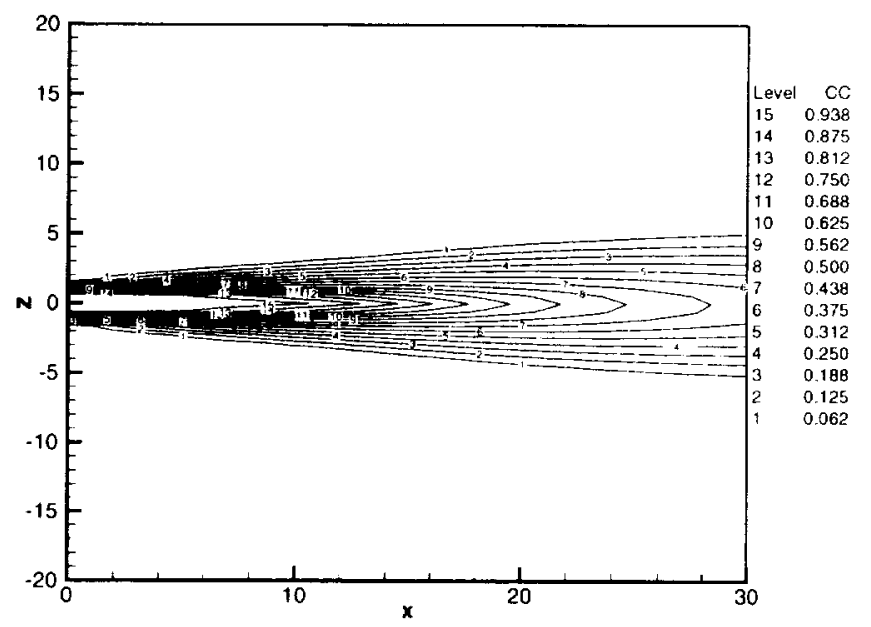

)

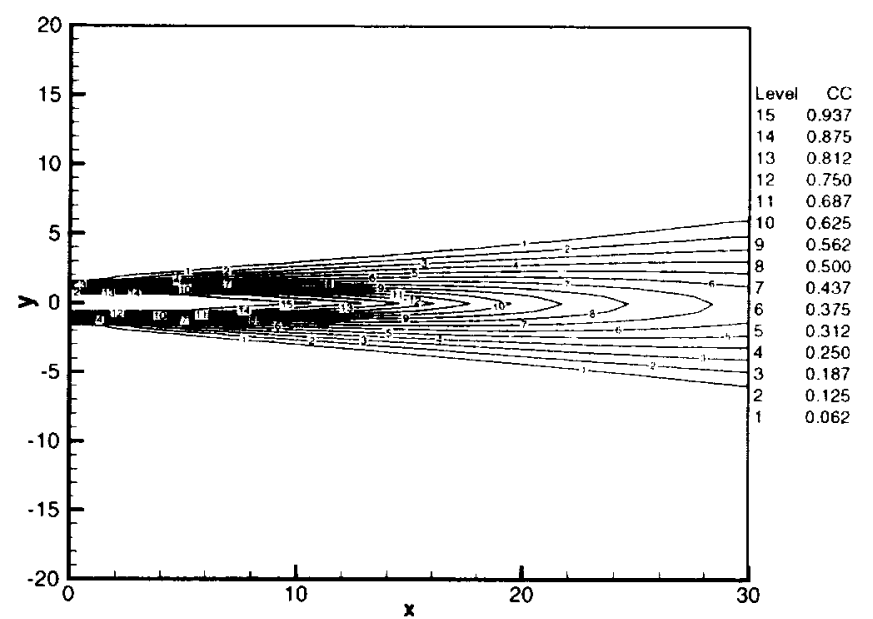

d

Fia. 8. Distributed configuration of two peripheral nozzles with parallel jets: (a,c)-axial velocity; (b,d)-concentration of particles; Jets" plane $(\mathrm{r}, z): a, b$. Middle plane $(x, y): c, d$ 


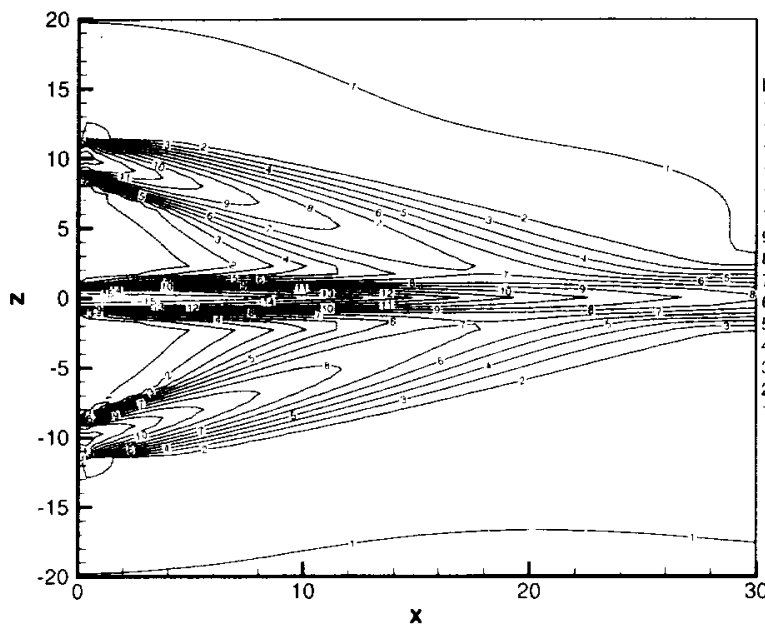

a

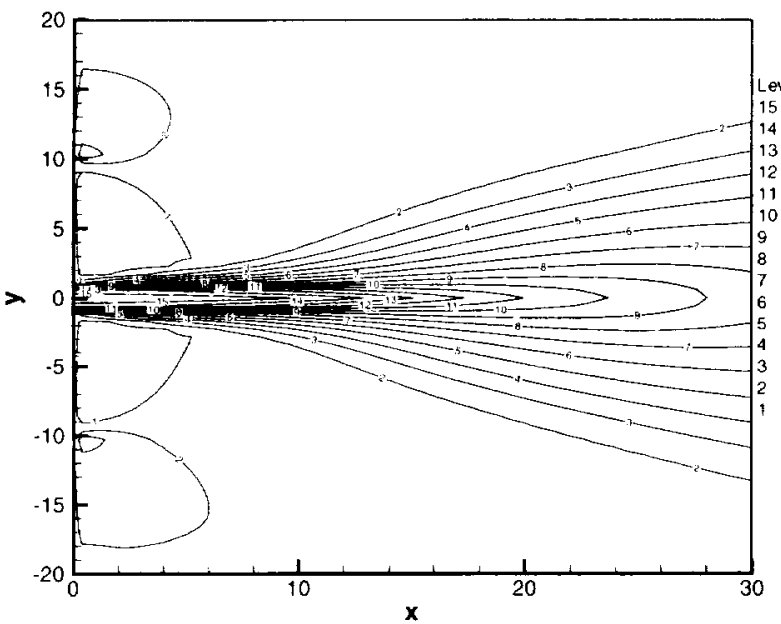

c:

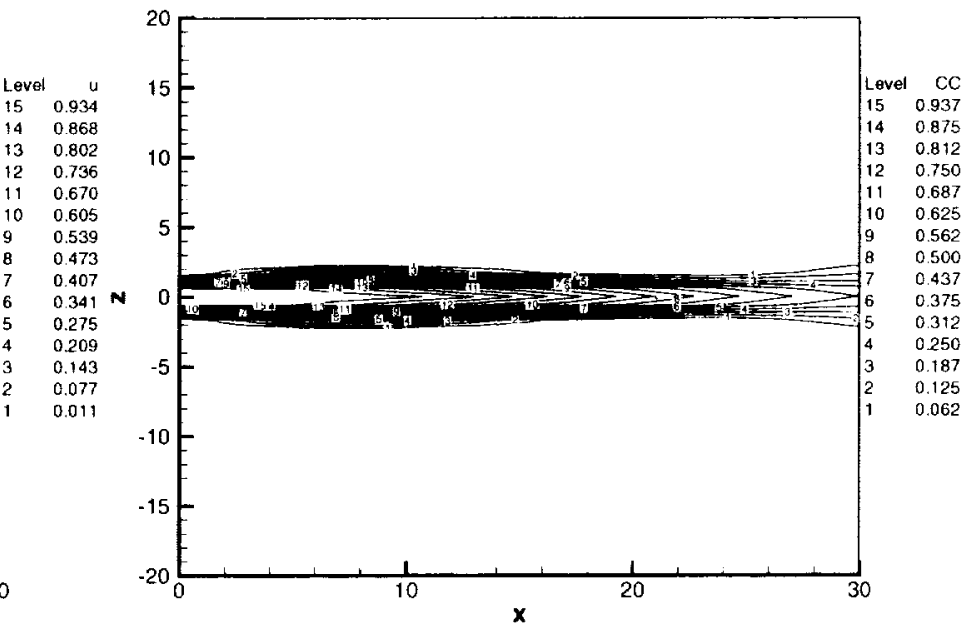

b

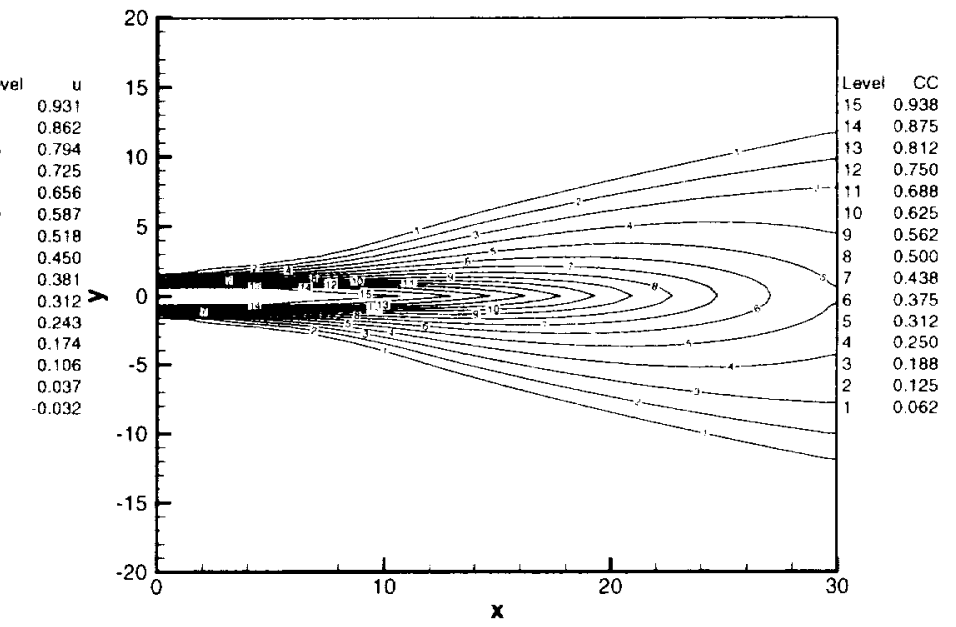

d

Fik. 9. Distributed configuration of two peripheral nozzles with $\alpha=30^{\circ}:(a, b)$ cross-section $(x, z)$ with peripheral jets: (c,d) (ross-section $(y, z)$ between peripheral jets; $(a, c)$-axial velocity; $(b, d)$-concentration of particles 


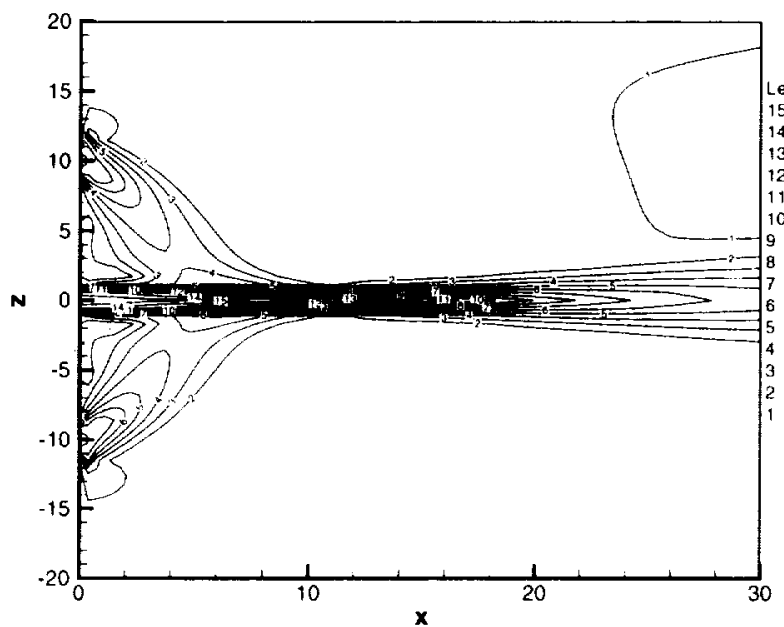

a

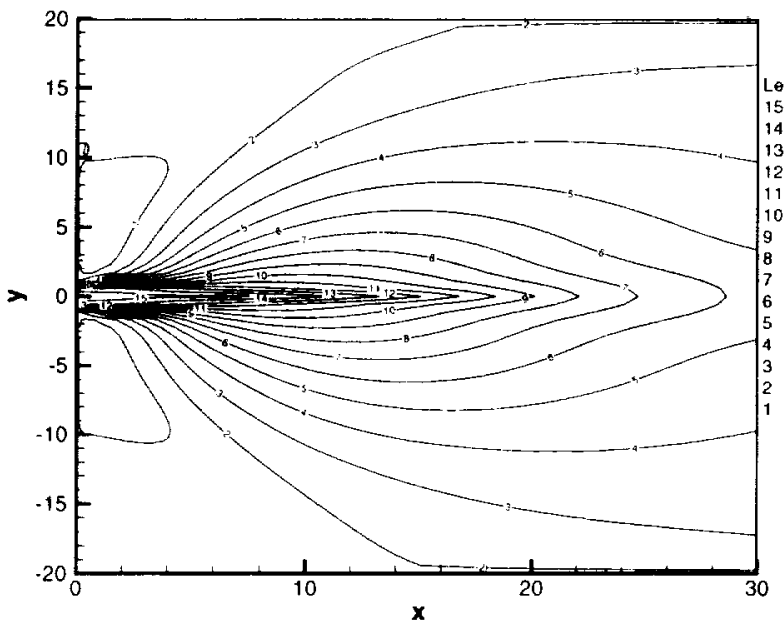

("

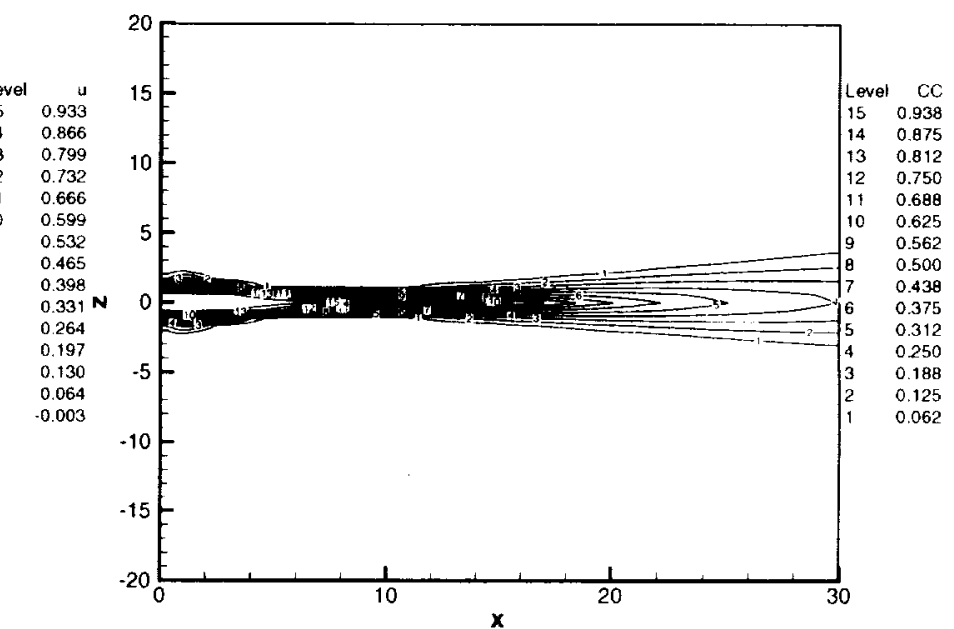

b

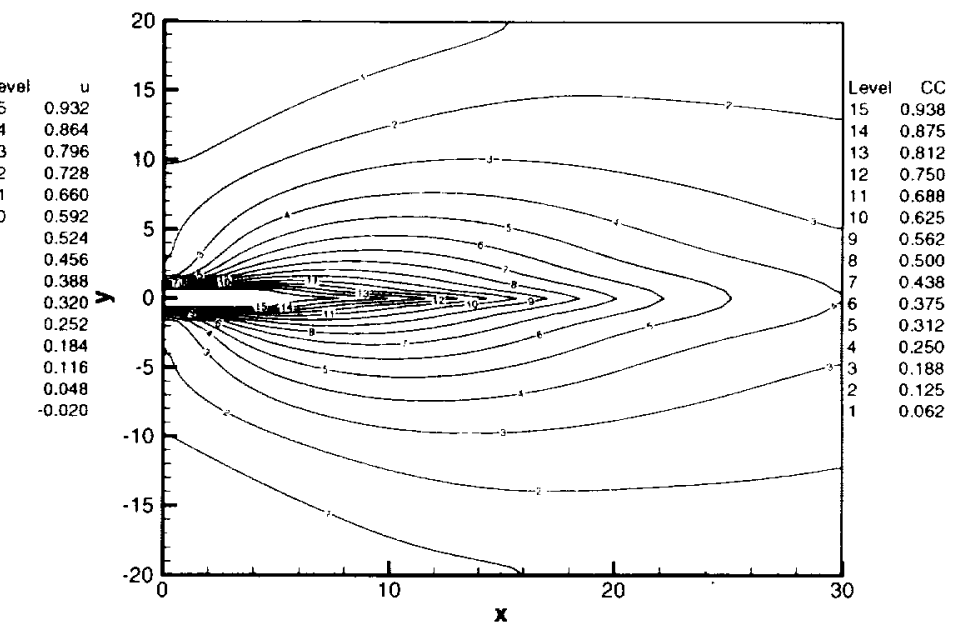

d

Fic. 10. Distributed configuration of two peripheral nozzles with $\alpha=60^{\circ}:(a, c)$-axial velocity; $(b, d)$-concentration of particles 


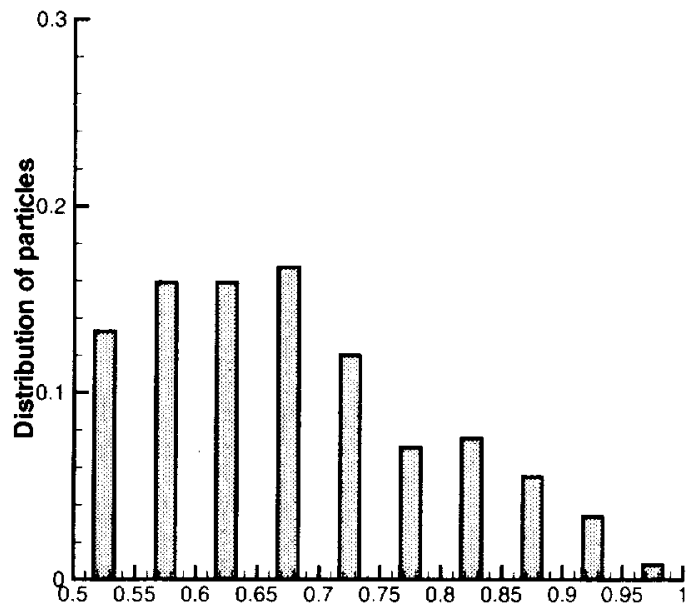

a. Normalized temperature, (T-Tjet)/(Tenv-Tjet)

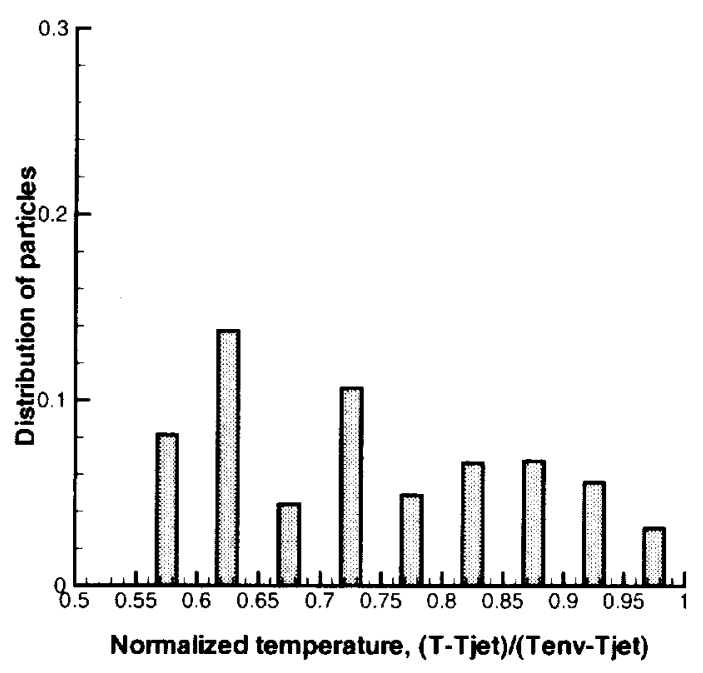

b)

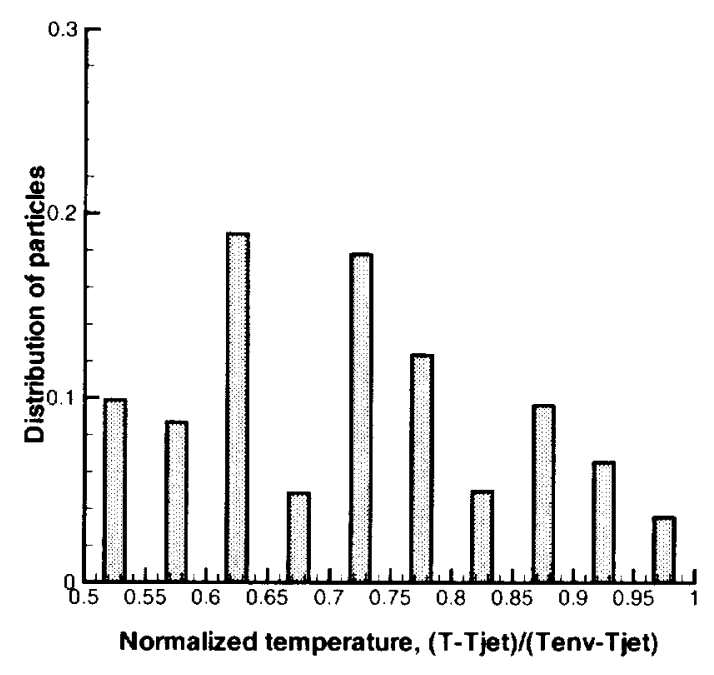

c 5, f) Gase 6 .
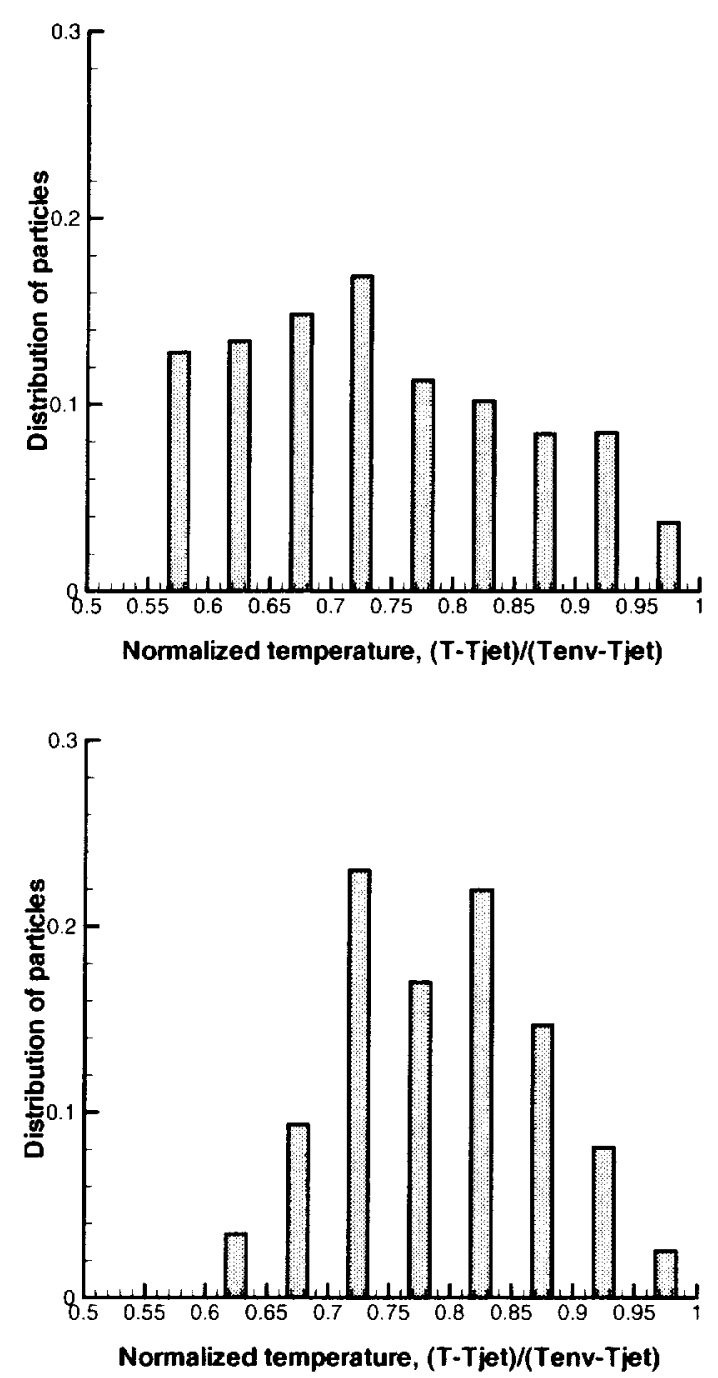

e

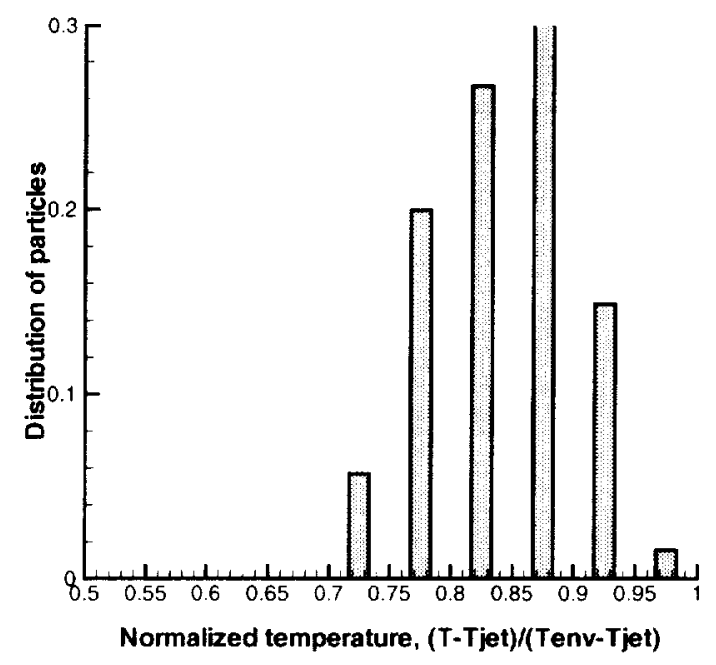

f 


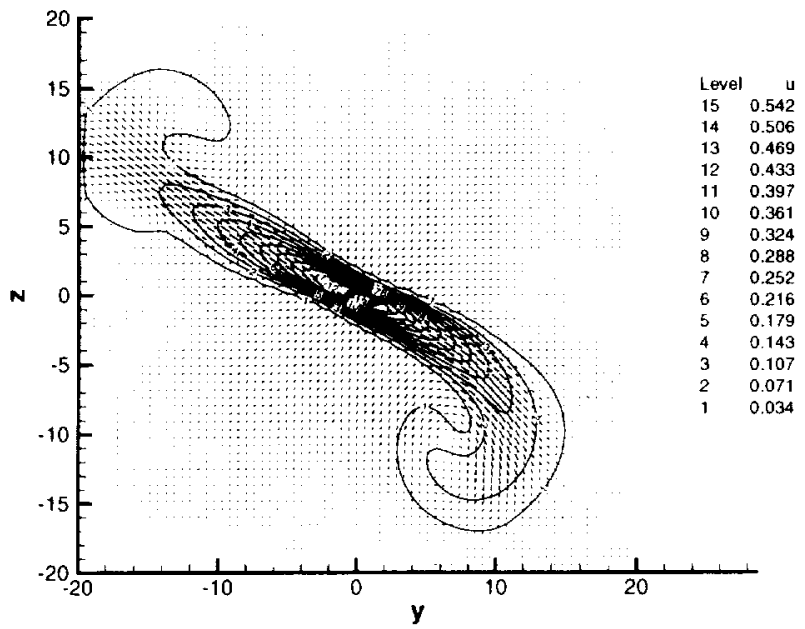

a

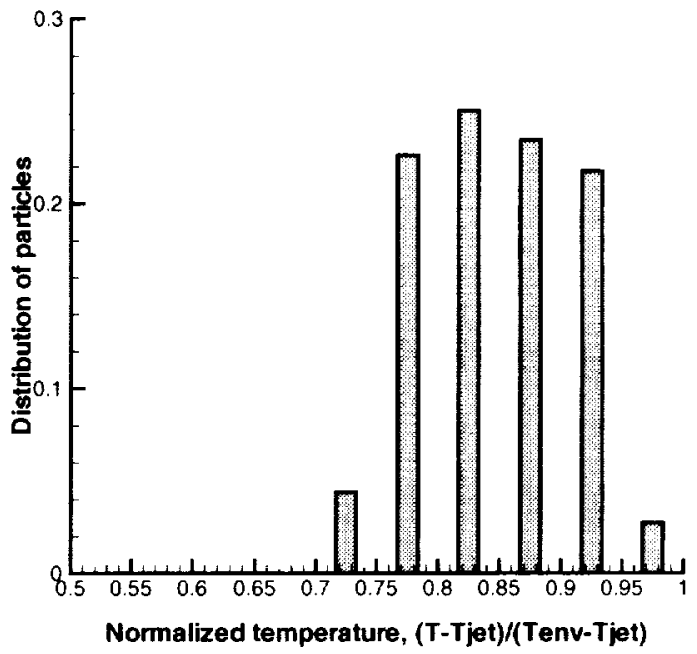

b

FIG. 12. Arrangement of peripheral jets with non-zero total angular momentum (Case 8). a) isolines of axial velocity and vectors $\left(l_{2}, \ell_{3}\right)$ at cross-section $\left.x=17.25 r_{0}, b\right)$ particle distribution by temperature at exit cross-section. 


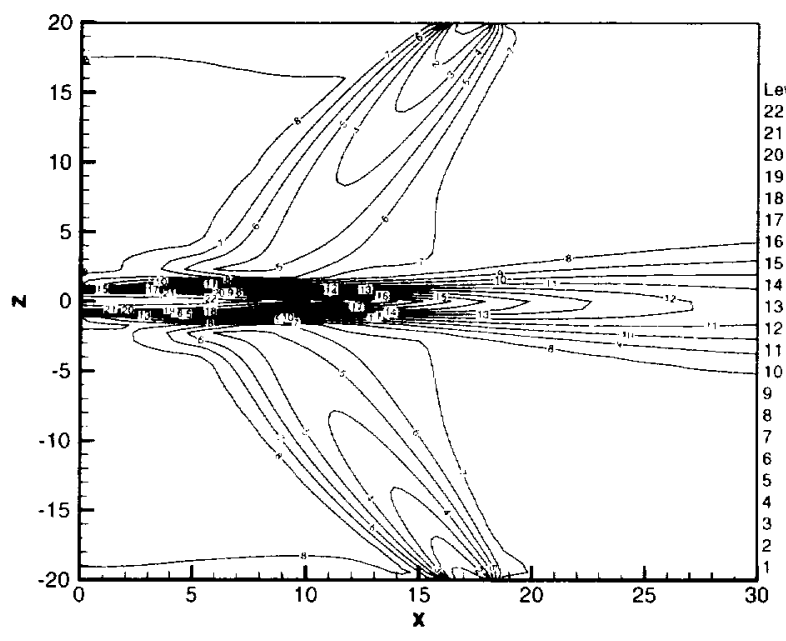

a

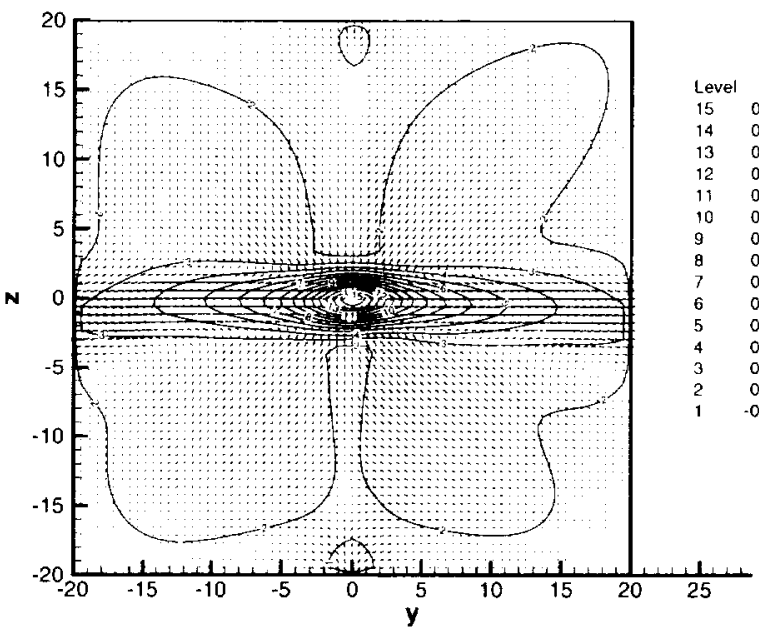

(c.

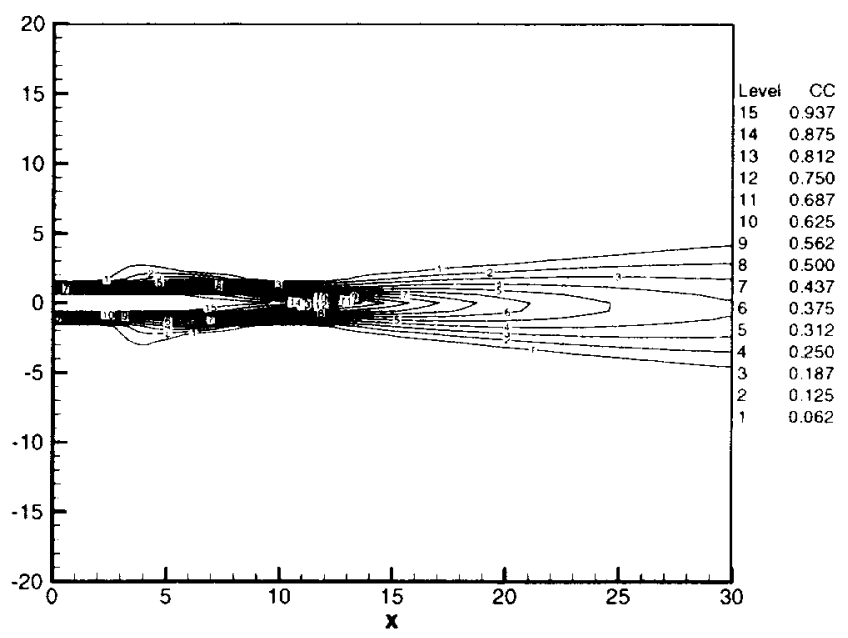

b)

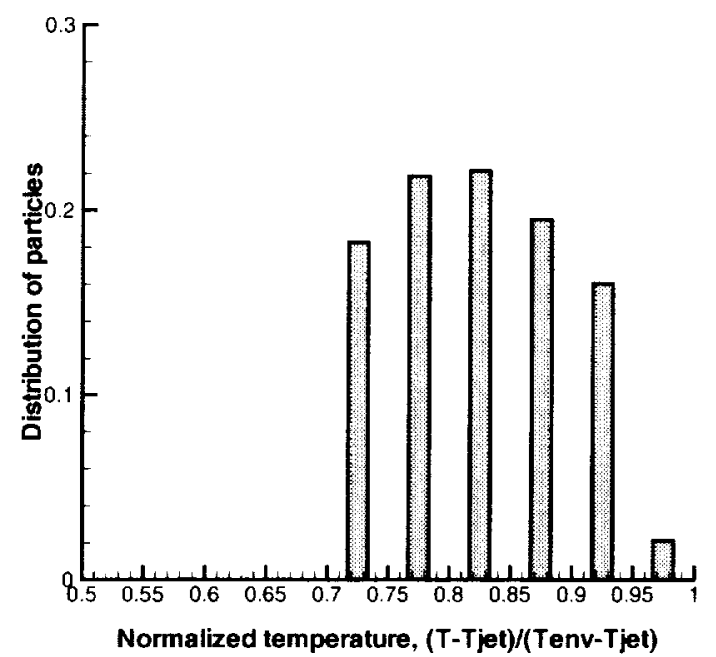

d

FiG. 13. Peripheral nozzles are drilled through the side walls (Case 9). (a,b) axial velocity and concentration at ( $x, z)$ section, c) isolines of axial velocity and vectors $\left(J_{2}, V_{3}\right)$ at cross-section $x=19.1 r_{0}$, d) particle distribution by temperature at cxit cross-section. 


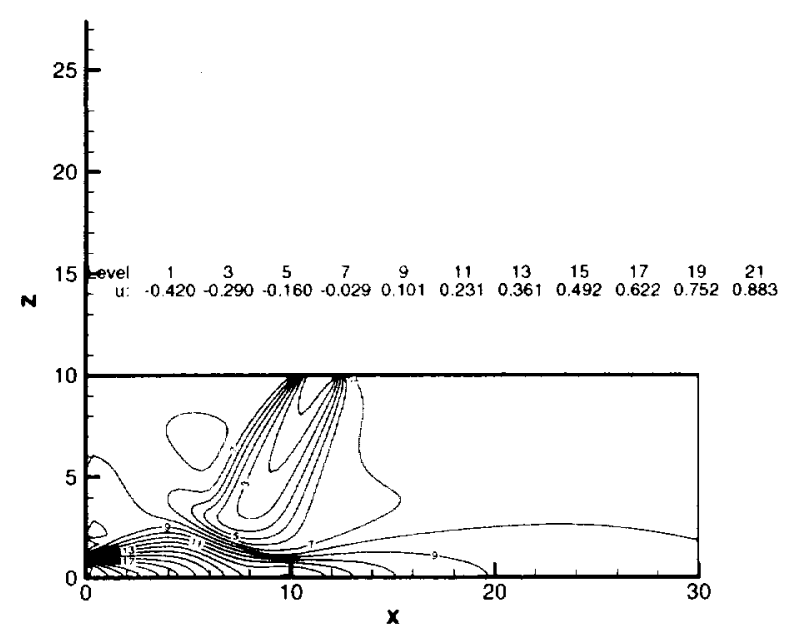

a

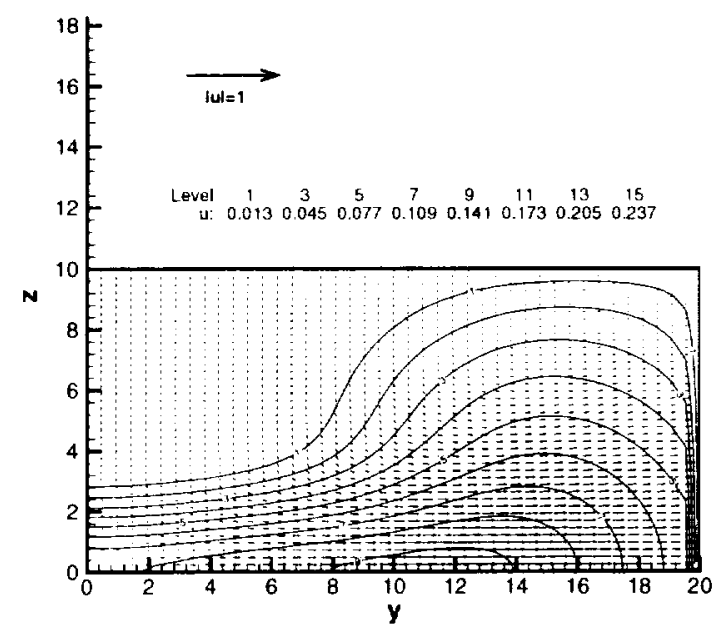

(c

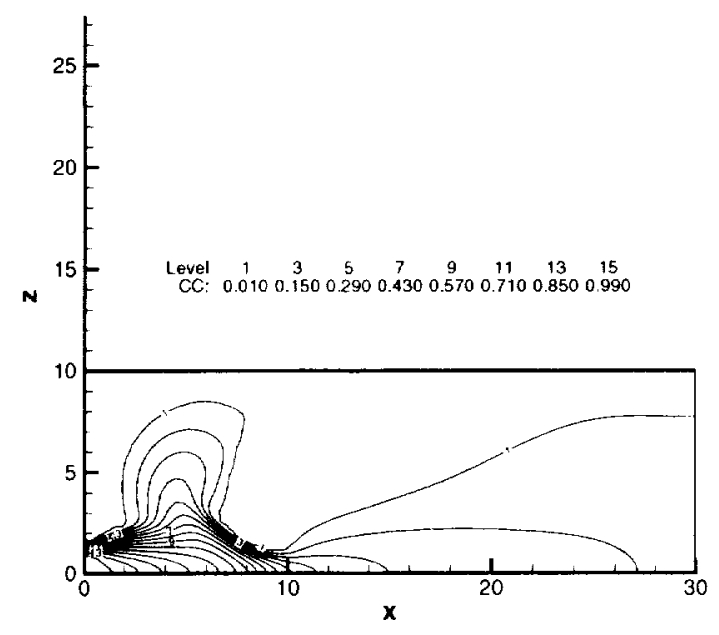

b)

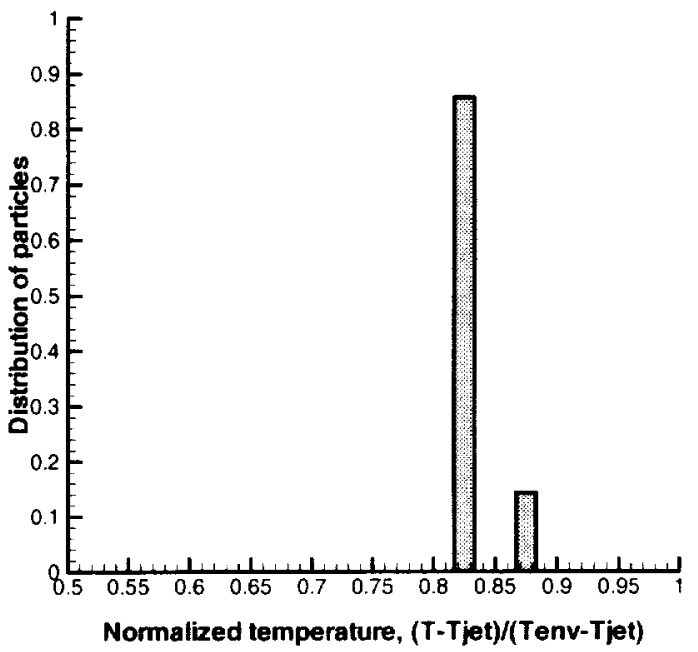

d

FI(i. 1.1. Rectangular cross-section of reactor (Case 10). (a,b) asial velocity and concentration at ( $x, z)$ section, c) isolines of axial velocity and vectors $\left(V_{2}, U_{3}\right)$ at cross-section $x=19.1 r_{0}$, d) particle distribution by temperature at exit cross-section. 


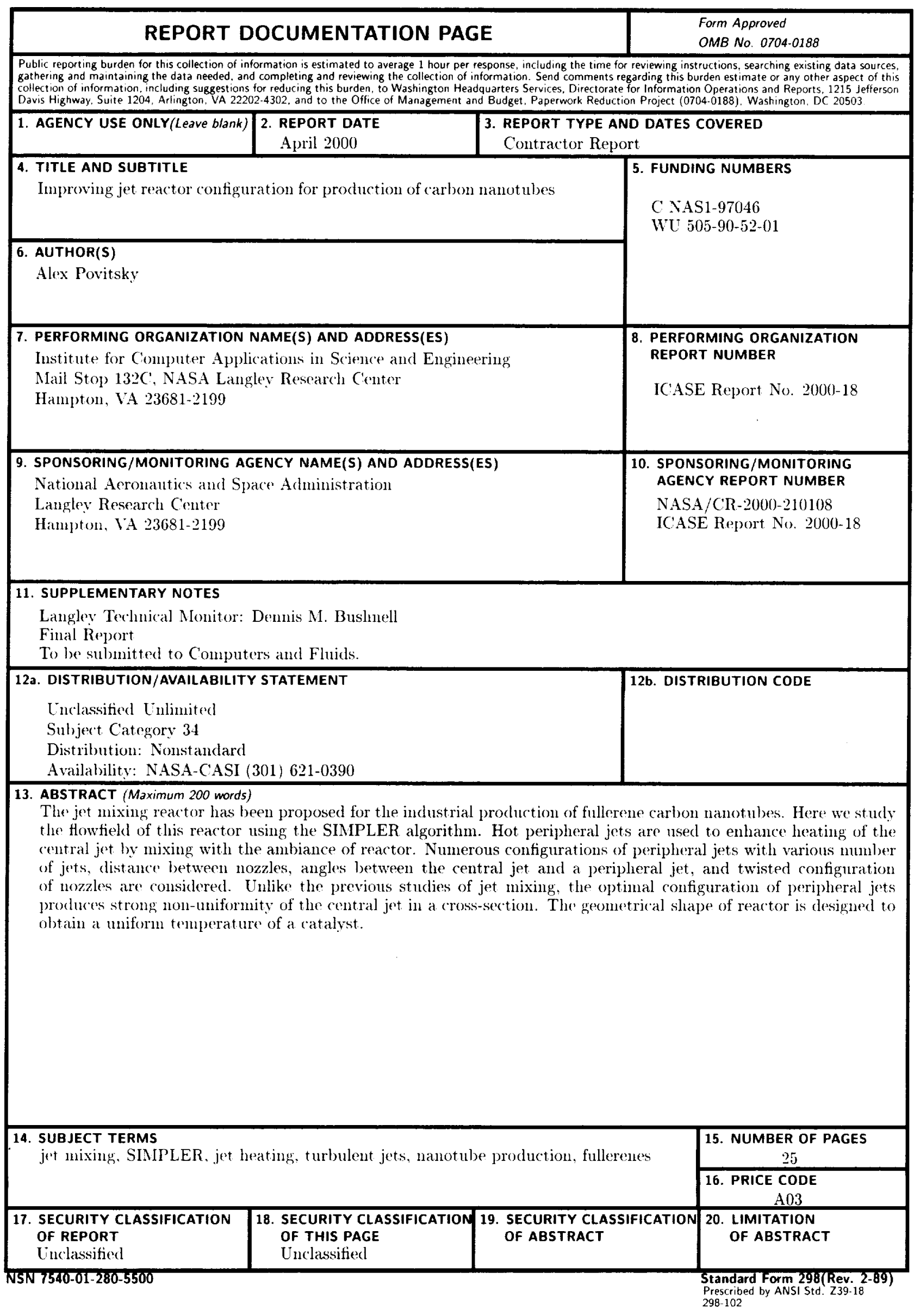



